\title{
Solubility of NaHCO3 inSystems of Aqueous Glycols (Monoethylene Glycol, Diethylene Glycol,Triethylene Glycol, and Tetraethylene Glycol): The Role of Solvation
}

\author{
Neerup, Randi; Villadsen, Sebastian N. B.; Thomsen, Kaj; Fosbøl, Philip L.
}

Published in:

Journal of Chemical and Engineering Data

Link to article, DOI:

10.1021/acs.jced.0c00538

Publication date:

2021

Document Version

Peer reviewed version

Link back to DTU Orbit

Citation (APA):

Neerup, R., Villadsen, S. N. B., Thomsen, K., \& Fosbøl, P. L. (2021). Solubility of $\mathrm{NaHCO}_{\text {inSystems of }}$

Aqueous Glycols (Monoethylene Glycol, Diethylene Glycol,Triethylene Glycol, and Tetraethylene Glycol): The Role of Solvation. Journal of Chemical and Engineering Data, 66(1), 222-233.

https://doi.org/10.1021/acs.jced.0c00538

\section{General rights}

Copyright and moral rights for the publications made accessible in the public portal are retained by the authors and/or other copyright owners and it is a condition of accessing publications that users recognise and abide by the legal requirements associated with these rights.

- Users may download and print one copy of any publication from the public portal for the purpose of private study or research.

- You may not further distribute the material or use it for any profit-making activity or commercial gain

- You may freely distribute the URL identifying the publication in the public portal 


\title{
Solubility of $\mathrm{NaHCO}_{3}$ in systems of aqueous glycols
}

\author{
(monoethylene glycol, diethylene glycol, triethylene
}

\author{
glycol, and tetraethylene glycol): The role of
}

\author{
Solvation
}

\author{
Randi Neerup, Sebastian N. B. Villadsen, Kaj Thomsen, Philip L. Fosbøl*
}

Center for Energy Resources Engineering (CERE), Department of Chemical and Biochemical Engineering, Technical University of Denmark (DTU), Søltofts Plads, building 229, 2800 Kgs.

Lyngby, Denmark

\begin{abstract}
:
The solubility and density of $\mathrm{NaHCO}_{3}$ in the systems: monoethylene glycol (MEG)-water, diethylene glycol (DEG)-water, triethylene glycol (TEG)-water, and tetraethylene glycol (TeEG)water have been measured over the entire concentration range from pure water to pure glycol. Experimental measurements have been conducted at the temperatures $5,20,40$, and $60{ }^{\circ} \mathrm{C}$, and at atmospheric pressure.
\end{abstract}

The solubility of $\mathrm{NaHCO}_{3}$ in all glycol mixtures were increasing with increasing temperatures. Solubility is largest in aqueous MEG and decreases with the higher molecular weights of glycol 
used. Local minima were observed in all systems in the concentration range 80 to $90 \mathrm{wt} \%$ glycol, salt-free. On a mole fraction scale, minima were found at $x$ (glycol, salt-free $)=0.5$. There is a clear tendency that two solvate mechanisms exist with a 1:1 solvation relation. One type of solvation is formed in $\mathrm{NaHCO}_{3}$-water, and a second type is formed in $\mathrm{NaHCO}_{3}$-glycol.

The density results showed a linearly increase up to a glycol concentration $\approx 80 \mathrm{wt} \%$. Above 80 $\mathrm{wt} \%$ the density seemed to form a minima similar to the solubility phase diagrams.

KEYWORDS: glycols, $\mathrm{NaHCO}_{3}$, solubility, corrosion, gas drying, gas hydrates

\section{Introduction}

Formation of gas hydrates and $\mathrm{CO}_{2}$ corrosion in wells, and pipelines are considered a major problem in the oil and gas industry ${ }^{1-5}$. It has been a recognised topic since the discovery in 1934 by Hammerschmidt ${ }^{6}$. Gas hydrate formation can cause serious plugs and lead to costly shut downs in the production line. In addition, it also creates a safety concern. Several methods for reducing/preventing gas hydrates have been considered, including reduction of the water content, changing operational conditions, and addition of additives also known as hydrate inhibitors ${ }^{7}$. Addition of hydrate inhibitors change the chemical potential of hydration towards lower temperature and higher pressure ${ }^{8}$. Usually, hydrate inhibitors as methanol, ethanol, sodium chloride, monoethylene glycol (MEG), and diethylene glycol (DEG) have been used ${ }^{7-9}$. The use of DEG for dehydrating natural gas has been employed since 1936 and later triethylene glycol (TEG), and tetraethylene glycol (TeEG) have proved suitable for the purpose ${ }^{10}$. Most commonly, DEG has been used for low temperature dehydration and higher molecular weight glycols as TEG, and TeEG for temperatures above $10{ }^{\circ} \mathrm{C}^{10}$. 
In the production of oil and gas, water and $\mathrm{CO}_{2}$ are produced. Approximately, $25 \%$ of the corrosion failures are related to $\mathrm{CO}_{2}$ corrosion ${ }^{2}$. Despite alternative materials with high corrosion resistance, the industry is widely using carbon steel for process equipment and pipelines due to its cost effective characteristics ${ }^{2,3,5}$. Once the material is exposed to different environments, protective film layers are formed as a product of chemical and electrochemical reactions. The protective barrier towards corrosion depends on different parameters such as $\mathrm{CO}_{2}$ partial pressure, solution $\mathrm{pH}$, temperature, and type of steel material ${ }^{2,3,5}$. At sweet conditions where no $\mathrm{H}_{2} \mathrm{~S}$ is present, the main corrosion product is $\mathrm{FeCO}_{3}$. $\mathrm{FeCO}_{3}$ is known to create protective layers extending the life of the pipelines. Increasing $\mathrm{pH}$ between $6-8^{1,2}$ stabilises $\mathrm{FeCO}_{3}$. $\mathrm{pH}$ stabilisers such as ammonia, sodium carbonate, and sodium bicarbonate have been used and have proven to reduce the corrosion rate significantly $y^{2,3,9}$.

Detailed knowledge of the solubility of bicarbonate in aqueous glycol systems are important for corrosion modelling of e.g. pipelines. The solubility of $\mathrm{NaHCO}_{3}$ in $\mathrm{MEG}-\mathrm{H}_{2} \mathrm{O}$ and in DEG- $\mathrm{H}_{2} \mathrm{O}$ have been investigated by several authors ${ }^{4,9,11-13}$, whereas none have considered the solubility of $\mathrm{NaHCO}_{3}$ in triethylene glycol (TEG)- $\mathrm{H}_{2} \mathrm{O}$ and in tetraethylene glycol (TeEG)- $\mathrm{H}_{2} \mathrm{O}$. Fosbøl et al. ${ }^{11}$ reported new data points for solubility and density in the mixed solvent $\mathrm{NaHCO}_{3}-\mathrm{MEG}-\mathrm{H}_{2} \mathrm{O}$ in the temperature range 2 to $60{ }^{\circ} \mathrm{C}$. They observed a minimum solubility at around $85 \mathrm{wt} \%$ MEG for all isotherms. X-ray powder diffraction (XRPD) analysis showed that the solid phase was $\mathrm{NaHCO}_{3}$ at all MEG concentrations. Gärtner et al. ${ }^{12}$ measured the solubility of $\mathrm{NaHCO}_{3}$ in the MEG- $\mathrm{H}_{2} \mathrm{O}$ system over the temperature range 50 to $90{ }^{\circ} \mathrm{C}$. They also observed a minimum solubility around 80 to $90 \mathrm{wt} \%$ MEG (salt-free). Sandengen ${ }^{13}$ measured the solubility of $\mathrm{NaHCO}_{3}$ in 60 and $90 \mathrm{wt} \%$ MEG at 4.0 and $21.9{ }^{\circ} \mathrm{C}$. Only at $4.0^{\circ} \mathrm{C}$ a solid phase of $\mathrm{NaHCO}_{3}$ was present. He concluded that equilibrium had not been reached. Dugstad and Seiersten ${ }^{9}$ measured few data 
points in the systems: $\mathrm{NaHCO}_{3}-\mathrm{MEG}-\mathrm{H}_{2} \mathrm{O}$ and in the $\mathrm{NaHCO}_{3}-\mathrm{DEG}-\mathrm{H}_{2} \mathrm{O}$. They studied the salt solubility in pure and in $90 \mathrm{wt} \%$ glycol mixtures and at the temperatures 20 and $124{ }^{\circ} \mathrm{C}$.

The aim of this work is to provide solubility and density data of the ternary systems: $\mathrm{NaHCO}_{3}-$ MEG- $\mathrm{H}_{2} \mathrm{O}, \mathrm{NaHCO}_{3}-\mathrm{DEG}-\mathrm{H}_{2} \mathrm{O}, \mathrm{NaHCO}_{3}-\mathrm{TEG}-\mathrm{H}_{2} \mathrm{O}$ and $\mathrm{NaHCO}_{3}-\mathrm{TeEG}_{2}-\mathrm{H}_{2} \mathrm{O}$. Furthermore, this work also aims to support thermodynamic modelling of corrosion and gas drying. These data are also useful for generic electrolyte thermodynamics and molecular theory development.

\section{Experimental details}

\subsection{Materials}

The studied chemicals are listed in Table 1. The chemicals were used as received without any further purification. Chemicals were mixed with demineralized water, which had a conductivity of $0.2 \mu \mathrm{S}$. Standardized $\mathrm{HCl}(0.1 \mathrm{M})$ used for titration was supplied from J. T. Baker.

Table 1. Chemicals used in the experiments.

\begin{tabular}{llccc}
\hline Chemical name & Source & CAS No. & Mass fraction purity & Purification method \\
\hline Diethylene glycol & Fluka & $111-46-6$ & $\geq 99.0$ & none \\
Triethylene glycol & Fluka & $112-27-6$ & $\geq 99.0$ & none \\
Monoethylene glycol & Riedel-deHaën & $107-21-1$ & $\geq 99.0$ & none \\
Tetraethylene glycol & Sigma-Aldrich & $112-60-7$ & $\geq 99.0$ & none \\
Sodium hydrogen carbonate & MERCK & $144-55-8$ & $>99.7$ & none \\
\hline
\end{tabular}

\subsection{Experimental method}

Solubility measurements were conducted equivalent to the method outlined by Fosbøl et al. ${ }^{4,11,14}$.

The equilibrium experiments were performed at atmospheric conditions at the temperatures 5, 20, 40 , and $60{ }^{\circ} \mathrm{C}$.

The setup consisted of an equilibrium unit with seven cells connected in parallel to a heating/cooling bath (Julabo F25). The heating/cooling bath controlled the temperature within 
$\pm 0.01{ }^{\circ} \mathrm{C}$. Each cell had a glass lid and a heat jacket surrounding an equilibrium chamber. Supersaturated $\mathrm{NaHCO}_{3}$ mixtures were added to $50 \mathrm{~mL}$ closed polyethylene (PE) containers, each containing a magnetic stirrer. The PE containers were placed in the equilibrium chamber.

The supersaturated mixtures were stirred at approximately 3-500 rpm and equilibrated over a period of 24 to $120 \mathrm{~h}$. The equilibration time needed for the analysis is short, but the time used was fixed to 24 hours on normal working days and 120 hours over weekends. The saturated liquid samples were filtered before analysis. A sample volume of $100-1000 \mathrm{~mL}$ was obtained by micropipette, taken in triplicate from each equilibrium chamber. Each sample volume was weighed and the density was determined as weight per volume. The accuracy of the density measurements is in the order of $0.01 \mathrm{~g} / \mathrm{cm}^{3}$. Less than 5 minutes after filtering the samples they were analyzed by potentiometric titration using an autoburette (Radiometer, ABU93 triburette). The software used to control the titration unit and the $\mathrm{pH}$ measurement was developed in-house. The accuracy of the equivalence points is approximately $0.01 \mathrm{~mL}$. Approximately, $15 \mathrm{~g}$ of demineralized water was added to the samples and titrated with $0.1 \mathrm{M} \mathrm{HCl}$. The initial $\mathrm{pH}$ before the potentiometric titration is in the order of 9-11.

\section{Results and discussion}

The solubility of $\mathrm{NaHCO}_{3}$ in the solvents: MEG- $\mathrm{H}_{2} \mathrm{O}$, DEG- $\mathrm{H}_{2} \mathrm{O}$, TEG- $\mathrm{H}_{2} \mathrm{O}$, and TeEG- $\mathrm{H}_{2} \mathrm{O}$ and the density of the mixtures were determined at atmospheric pressure, and at the temperatures 5, 20, 40, and $60^{\circ} \mathrm{C}$. Experimental results, such as initial compositions, titration results, densities, the compositions of the saturated solutions, and the composition of the solid phase are presented in Table 2, Table 3, Table 4, and Table 5. The compositions of the saturated solutions and the solid phase were determined by the reverse Schreinemakers (RS) method previously explained ${ }^{14}$. The 
composition is denoted $S_{T N a}$ and is given as the total amount of sodium (moles of Na) per $\mathrm{kg}$ of solution. Experimental results are given as an average of three measurements together with standard deviations. The results obtained from the RS method are very accurate as standard deviations are low. The uncertainty given in Table 2, Table 3, Table 4, and Table 5 is an expression of the uncertainty of the triple titration during compositional analysis. The uncertainty does not include deviations caused by slight temperature differences, filtering efficiency, titrant standardization, sampling accuracy etc. The real uncertainty is most likely 10 times higher. This can be observed by plotting solubilities as function of temperature in the range of the minimum observed.

At higher temperatures, the $\mathrm{NaHCO}_{3}$ solution has a tendency to break down into $\mathrm{NaOH}$ and $\mathrm{CO}_{2}$. For high temperature solubility analysis of $\mathrm{NaCO}_{3}$, this break down needs to be taken into account. Our analysis has shown ${ }^{14}$, that the $S_{T N a}$ number very trust worthy independent of evaporated $\mathrm{CO}_{2}$ content.

The solubility of $\mathrm{NaHCO}_{3}$ in the aqueous glycol mixtures are graphically presented in Figure 1, Figure 2, Figure 3, and Figure 4. For all systems, a moderate solubility decline with increased glycol concentrations was observed until minima between 80 to $90 \mathrm{wt} \%$ were reached. Hereafter a gradual increase in the solubility at higher glycol content is seen. This behaviour is also seen in $\mathrm{CaSO}_{4}-\mathrm{MEG}-\mathrm{H}_{2} \mathrm{O}$ and $\mathrm{CaCO}_{3}-\mathrm{MEG}-\mathrm{H}_{2} \mathrm{O}^{15}$. This phenomenon was studied and validated for the $\mathrm{NaHCO}_{3}-\mathrm{MEG}-\mathrm{H}_{2} \mathrm{O}$ system by Fosbøl et al. ${ }^{11}$. In the study, the identity of the solid phase as pure $\mathrm{NaHCO}_{3}$ was confirmed by XRPD. Fosbøl et al. ${ }^{11}$ proposed that the increased solubility could be explained by the increase of $\mathrm{CO}_{2}$ solubility at high MEG concentrations. Gärtner et al. ${ }^{12}$ suggested that the increased $\mathrm{NaHCO}_{3}$ solubility could be due to soluble complexes of MEG with $\mathrm{HCO}_{3}^{-}$. In this study, XRPD was only applied to the system $\mathrm{NaHCO}_{3}-\mathrm{MEG}-\mathrm{H}_{2} \mathrm{O}$ and not to the systems 
containing DEG, TEG, and TeEG. Precipitates may therefore be complexes of glycol and $\mathrm{HCO}_{3}^{-}$ . A further study on the precipitates using either Raman and/or infrared (IR) spectroscopy would be valuable.

The solubility of $\mathrm{NaHCO}_{3}$, in all glycols, is increasing by increasing the temperature. However, the temperature dependency decreases towards higher glycol concentrations. At glycol (salt-free) concentrations above $70 \mathrm{wt} \%$ the $\mathrm{NaHCO}_{3}$ solubility is more or less identical comparing the results at the different temperatures.

Results obtained in this study for $\mathrm{NaHCO}_{3}-\mathrm{MEG}-\mathrm{H}_{2} \mathrm{O}$ (see Figure 1) are in line with the data published by Fosbøl ${ }^{4}$, and Fosbøl et al. ${ }^{11}$ at the temperatures 20,40 , and $60{ }^{\circ} \mathrm{C}$. The minima are located approximately at $w(\mathrm{MEG}) \approx 85 \%$ and $w\left(\mathrm{NaHCO}_{3}\right) \approx 3 \%$ corresponding to the findings of Fosbøl et al. ${ }^{4,11}$.

The solubility trend of $\mathrm{NaHCO}_{3}$ in $\mathrm{DEG}-\mathrm{H}_{2} \mathrm{O}$, Figure 2, is similar to the solubility of $\mathrm{NaHCO}_{3}$ in $\mathrm{MEG}-\mathrm{H}_{2} \mathrm{O}$. The lowest value of the solubility is found in the range $85 \% \leq w(D E G) \leq 90 \%$. The minimum is more flat and the $\mathrm{NaHCO}_{3}$ increase is not as pronounced as with MEG. The minimum solubility of $\mathrm{NaHCO}_{3}$ is lower in TEG- $\mathrm{H}_{2} \mathrm{O}$ (Figure 3) and in TeEG- $\mathrm{H}_{2} \mathrm{O}$ (Figure 4) $w\left(\mathrm{NaHCO}_{3}\right.$ ) $\approx 1 \%$ and $w\left(\mathrm{NaHCO}_{3}\right) \approx 0.5 \%$ respectively. The minima in these glycols are shifted towards higher glycol concentrations, which makes the solubility increase less distinct.

Table 2. Experimental data of the system $\mathrm{NaHCO}_{3}-\mathrm{MEG}-\mathrm{H}_{2} \mathrm{O}$ measured at 5, 20, 40 and $60{ }^{\circ} \mathrm{C}$ and at a pressure $\mathrm{p}=0.1 \mathrm{MPa}^{\mathrm{a}}$. $t$ is the equilibrium temperature. $\widetilde{w}$ is the salt-free glycol concentration (wt\%). $\sigma$ is the standard deviation. The results of the RS method was calculated from equations of Fosbøl et al. ${ }^{4,14} \cdot w_{i}$ is the weight percent of component $i$. The solid phase is assumed from a subset of analysis and not every sample was characterized by PXRD. 


\begin{tabular}{|c|c|c|c|c|c|c|c|c|c|c|c|c|c|}
\hline \multirow{3}{*}{$\begin{array}{c}\mathrm{t} \\
{ }^{\circ} \mathrm{C} \\
\end{array}$} & \multicolumn{4}{|c|}{ initial } & \multicolumn{4}{|c|}{ equilibrium } & \multicolumn{5}{|c|}{ reverse Schreinemakers } \\
\hline & $\widetilde{w}_{M E G}$ & $m_{\mathrm{H}_{2} \mathrm{O}}$ & $m_{M E G}$ & & $S_{T N a}$ & $\sigma_{S_{T N a}}$ & $\begin{array}{l}\rho_{\text {sat,liq }} \\
\mathrm{g} / \mathrm{cm}\end{array}$ & $\sigma_{\rho}$ & $w_{\mathrm{NaHCO}_{3}}$ & $\sigma_{\mathrm{NaHCO}_{3}}$ & $m_{\mathrm{H}_{2} \mathrm{O}}$ & $w_{M E G}$ & $\mathrm{P}$ \\
\hline & $\%$ & $\mathrm{~g}$ & g & $\mathrm{g}$ & $\mathrm{mol} / \mathrm{kg}$ & $\mathrm{mol} / \mathrm{kg}$ & 3 & $\mathrm{~g} / \mathrm{cm}^{3}$ & $\%$ & $\%$ & $\mathrm{~g}$ & $\%$ & \\
\hline 5.20 & 0.00 & 10.045 & 0.000 & 1.003 & 0.869 & 0.006 & 1.005 & 0.016 & 7.30 & 0.054 & 10.045 & 0.00 & B \\
\hline 5.10 & 3.32 & 9.742 & 0.335 & 1.006 & 0.825 & 0.003 & 1.012 & 0.017 & 6.93 & 0.023 & 9.742 & 3.09 & B \\
\hline 5.00 & 15.05 & 8.502 & 1.506 & 0.950 & 0.690 & 0.005 & 1.005 & 0.014 & 5.80 & 0.046 & 8.502 & 14.17 & B \\
\hline 5.05 & 28.04 & 7.201 & 2.806 & 0.804 & 0.585 & 0.006 & 1.034 & 0.015 & 4.91 & 0.051 & 7.201 & 26.66 & B \\
\hline 5.50 & 53.00 & 4.725 & 5.328 & 0.745 & 0.373 & 0.0009 & 1.079 & 0.011 & 3.13 & 0.008 & 4.725 & 51.34 & B \\
\hline 5.20 & 78.00 & 2.205 & 7.815 & 0.601 & 0.292 & 0.002 & 1.108 & 0.006 & 2.45 & 0.013 & 2.205 & 76.08 & B \\
\hline 5.10 & 84.89 & 1.515 & 8.511 & 0.718 & 0.299 & 0.002 & 1.115 & 0.012 & 2.51 & 0.017 & 1.515 & 82.76 & B \\
\hline 5.05 & 89.96 & 1.006 & 9.013 & 0.805 & 0.324 & 0.0004 & 1.137 & 0.013 & 2.72 & 0.003 & 1.006 & 87.52 & B \\
\hline 5.10 & 94.66 & 0.536 & 9.511 & 1.055 & 0.392 & 0.0006 & 1.112 & 0.018 & 3.30 & 0.005 & 0.536 & 91.54 & B \\
\hline 5.50 & 100 & 0.000 & 10.007 & 1.009 & 0.610 & 0.0004 & 1.165 & 0.019 & 5.12 & 0.003 & 0.000 & 94.88 & $\mathrm{~B}$ \\
\hline 20.1 & 0.00 & 9.997 & 0.000 & 1.365 & 1.090 & 0.005 & 1.059 & 0.007 & 9.16 & 0.046 & 9.9972 & 0.00 & B \\
\hline 20.10 & 15.46 & 8.494 & 1.554 & 1.237 & 0.845 & 0.002 & 1.065 & 0.001 & 7.10 & 0.016 & 8.494 & 14.36 & B \\
\hline 20.10 & 28.03 & 7.232 & 2.816 & 1.112 & 0.687 & 0.005 & 1.078 & 0.004 & 5.77 & 0.043 & 7.232 & 26.41 & B \\
\hline 20.05 & 53.31 & 4.697 & 5.363 & 0.871 & 0.450 & 0.002 & 1.074 & 0.030 & 3.78 & 0.017 & 4.697 & 51.30 & B \\
\hline 20.10 & 78.05 & 2.218 & 7.890 & 0.754 & 0.350 & 0.005 & 1.107 & 0.017 & 2.94 & 0.043 & 2.218 & 75.76 & B \\
\hline 20.20 & 89.97 & 1.006 & 9.021 & 0.758 & 0.373 & 0.0009 & 1.130 & 0.006 & 3.13 & 0.008 & 1.006 & 87.15 & B \\
\hline 20.10 & 94.90 & 0.511 & 9.514 & 0.993 & 0.451 & 0.0007 & 1.135 & 0.001 & 3.79 & 0.006 & 0.511 & 91.30 & B \\
\hline 20.10 & 100.00 & 0.000 & 10.011 & 1.370 & 0.806 & 0.0003 & 1.157 & 0.002 & 6.77 & 0.002 & 0.000 & 93.23 & B \\
\hline 40.10 & 0.00 & 10.009 & 0.000 & 1.767 & 1.441 & 0.005 & 1.116 & 0.012 & 12.11 & 0.042 & 10.009 & 0.00 & B \\
\hline 40.20 & 15.09 & 8.510 & 1.513 & 1.628 & 1.129 & 0.013 & 1.069 & 0.052 & 9.48 & 0.108 & 8.510 & 13.66 & B \\
\hline 40.20 & 27.88 & 7.245 & 2.801 & 1.499 & 0.905 & 0.008 & 1.087 & 0.008 & 7.60 & 0.065 & 7.245 & 25.76 & B \\
\hline 40.20 & 52.90 & 4.730 & 5.313 & 1.241 & 0.579 & 0.006 & 1.083 & 0.006 & 4.87 & 0.051 & 4.73 & 50.33 & B \\
\hline 40.10 & 77.57 & 2.259 & 7.813 & 0.755 & 0.423 & 0.0004 & 1.137 & 0.003 & 3.55 & 0.003 & 2.259 & 74.81 & B \\
\hline 40.20 & 89.76 & 1.027 & 9.002 & 0.756 & 0.444 & 0.0005 & 1.144 & 0.010 & 3.73 & 0.004 & 1.027 & 86.42 & B \\
\hline 40.20 & 94.72 & 0.530 & 9.507 & 0.990 & 0.533 & 0.003 & 1.145 & 0.005 & 4.48 & 0.025 & 0.530 & 90.47 & B \\
\hline 40.20 & 100.00 & 0.000 & 10.019 & 1.366 & 0.913 & 0.001 & 1.177 & 0.005 & 7.67 & 0.009 & 0.000 & 92.33 & B \\
\hline 57.60 & 96.97 & 0.303 & 9.704 & 1.401 & 0.712 & 0.0008 & 1.176 & 0.006 & 5.98 & 0.006 & 0.303 & 91.18 & B \\
\hline 57.65 & 100.00 & 0.000 & 10.025 & 1.402 & 1.028 & 0.001 & 1.167 & 0.015 & 8.63 & 0.010 & 0.000 & 91.37 & B \\
\hline 59.50 & 15.05 & 8.512 & 1.508 & 1.410 & 1.480 & 0.004 & 1.162 & 0.034 & 12.43 & 0.034 & 8.512 & 13.18 & B \\
\hline 59.30 & 28.07 & 7.235 & 2.823 & 1.306 & 1.196 & 0.002 & 1.194 & 0.027 & 10.04 & 0.013 & 7.235 & 25.25 & B \\
\hline 59.80 & 0.00 & 10.011 & 0.000 & 2.206 & 2.008 & 0.02 & 1.148 & 0.005 & 16.86 & 0.171 & 10.011 & 0.00 & B \\
\hline 59.05 & 27.95 & 7.213 & 2.798 & 1.600 & 1.189 & 0.006 & 1.157 & 0.008 & 9.98 & 0.051 & 7.213 & 25.16 & B \\
\hline 59.30 & 42.94 & 5.742 & 4.321 & 1.502 & 0.911 & 0.008 & 1.148 & 0.041 & 7.65 & 0.068 & 5.742 & 39.65 & B \\
\hline 59.50 & 52.89 & 4.744 & 5.327 & 1.310 & 0.760 & 0.01 & 1.135 & 0.034 & 6.38 & 0.089 & 4.744 & 49.52 & B \\
\hline 59.45 & 67.97 & 3.211 & 6.815 & 1.245 & 0.589 & 0.0003 & 1.112 & 0.011 & 4.95 & 0.003 & 3.211 & 64.61 & B \\
\hline 59.10 & 77.98 & 2.210 & 7.827 & 0.707 & 0.522 & 0.0004 & 1.146 & 0.019 & 4.39 & 0.003 & 2.21 & 74.56 & B \\
\hline 59.35 & 78.54 & 1.507 & 5.515 & 0.804 & 0.510 & 0.0006 & 1.155 & 0.015 & 4.28 & 0.005 & 1.507 & 75.18 & B \\
\hline 59.45 & 89.88 & 1.014 & 9.008 & 0.916 & 0.533 & 0.001 & 1.150 & 0.023 & 4.48 & 0.010 & 1.014 & 85.85 & B \\
\hline 59.40 & 94.88 & 0.514 & 9.519 & 1.202 & 0.630 & 0.002 & 1.167 & 0.013 & 5.29 & 0.021 & 0.514 & 89.86 & $\mathrm{~B}$ \\
\hline
\end{tabular}

${ }^{a}$ Standard uncertainties $(u)$ for temperature, $\mathrm{wt} \%$, density, pressure, are $\mathrm{u}\left({ }^{\circ} \mathrm{C}\right)=0.01^{\circ} \mathrm{C}, \mathrm{u}(w)=$ $0.003+0.07, \mathrm{u}\left(S_{T N A}\right)=0.03 \mathrm{~mol} / \mathrm{kg}, \mathrm{u}(p)=1 \mathrm{kPa}$. Standard uncertainties of the densities are estimated as the standard deviations of the mean reported in the table. 
Table 3. Experimental data of the system $\mathrm{NaHCO}_{3}-\mathrm{DEG}-\mathrm{H}_{2} \mathrm{O}$ measured at 5, 20, 40 and $60{ }^{\circ} \mathrm{C}$ and at a pressure $\mathrm{p}=0.1 \mathrm{MPa}^{\mathrm{a}} . t$ is the equilibrium temperature. $\widetilde{w}$ is the salt-free glycol concentration (wt\%). $\sigma$ is the standard deviation. The results of the RS method was calculated from equations of Fosbøl et al. ${ }^{4,14} \cdot w_{i}$ is the weight percent of component $i$. The solid phase is assumed from a subset of analysis and not every sample was characterized by PXRD.

\begin{tabular}{|c|c|c|c|c|c|c|c|c|c|c|c|c|c|}
\hline \multirow[b]{2}{*}{$\begin{array}{c}\mathrm{t} \\
{ }^{\circ} \mathrm{C}\end{array}$} & \multicolumn{4}{|c|}{ initial } & \multicolumn{4}{|c|}{ equilibrium } & \multicolumn{5}{|c|}{ reverse Schreinemakers } \\
\hline & $\begin{array}{c}\widetilde{w}_{D E G} \\
\%\end{array}$ & $\begin{array}{c}m_{\mathrm{H}_{2} \mathrm{O}} \\
\mathrm{g}\end{array}$ & $\begin{array}{c}m_{D E G} \\
\mathrm{~g}\end{array}$ & $\begin{array}{c}m_{\mathrm{NaHCO}_{3}} \\
\mathrm{~g}\end{array}$ & $\begin{array}{c}S_{T N a} \\
\mathrm{~mol} / \mathrm{kg}\end{array}$ & $\begin{array}{c}\sigma_{S_{T N a}} \\
\mathrm{~mol} / \mathrm{kg}\end{array}$ & $\begin{array}{l}\rho_{\text {sat, liq }} \\
\mathrm{g} / \mathrm{cm}^{3}\end{array}$ & $\begin{array}{c}\sigma_{\rho} \\
\mathrm{g} / \mathrm{cm}^{3}\end{array}$ & $\begin{array}{c}w_{\mathrm{NaHCO}_{3}} \\
\%\end{array}$ & $\begin{array}{c}\sigma_{\mathrm{NaHCO}_{3}} \\
\%\end{array}$ & $\begin{array}{c}m_{\mathrm{H}_{2} \mathrm{O}} \\
\mathrm{g}\end{array}$ & $\begin{array}{c}w_{D E G} \\
\%\end{array}$ & $\mathrm{P}$ \\
\hline 5.2 & 0 & 10.004 & 0.000 & 1.515 & 0.87 & 0.002 & 1.021 & 0.009 & 7.31 & 0.018 & 10.004 & 0 & B \\
\hline 5.1 & 3.1 & 9.701 & 0.311 & 1.401 & 0.818 & 0.008 & 1.017 & 0.004 & 6.87 & 0.068 & 9.701 & 2.89 & B \\
\hline 5.05 & 15.17 & 8.528 & 1.525 & 1.307 & 0.626 & 0.014 & 1.033 & 0.007 & 5.26 & 0.119 & 8.528 & 14.38 & B \\
\hline 5.1 & 40.12 & 6.023 & 4.036 & 1.223 & 0.295 & 0.0008 & 1.068 & 0.01 & 2.48 & 0.006 & 6.023 & 39.13 & B \\
\hline 5.5 & 60 & 4.019 & 6.027 & 1.005 & 0.16 & 0.0003 & 1.096 & 0.007 & 1.35 & 0.003 & 4.019 & 59.19 & B \\
\hline 5.5 & 71.88 & 2.816 & 7.198 & 0.910 & 0.113 & 0.0002 & 1.098 & 0.008 & 0.95 & 0.002 & 2.816 & 71.19 & B \\
\hline 5.1 & 79.76 & 2.052 & 8.082 & 0.906 & 0.091 & 0.0002 & 1.108 & 0.01 & 0.76 & 0.002 & 2.052 & 79.15 & B \\
\hline 5.05 & 87.5 & 1.259 & 8.811 & 1.227 & 0.08 & 0.0001 & 1.112 & 0.01 & 0.67 & 0.001 & 1.259 & 86.91 & B \\
\hline 5.1 & 94.98 & 0.504 & 9.523 & 1.401 & 0.088 & 0.001 & 1.117 & 0.006 & 0.74 & 0.008 & 0.504 & 94.27 & B \\
\hline 5.2 & 100 & 0.000 & 10.050 & 1.509 & 0.163 & 0.0001 & 1.118 & 0.009 & 1.37 & 0.001 & 0.000 & 98.63 & B \\
\hline 19.8 & 0 & 10.004 & 0.000 & 1.500 & 1.095 & 0.0005 & 1.044 & 0.003 & 9.19 & 0.004 & 10.004 & 0 & B \\
\hline 19.8 & 3.39 & 9.724 & 0.341 & 1.400 & 1.013 & 0.0006 & 1.04 & 0.003 & 8.51 & 0.005 & 9.724 & 3.1 & B \\
\hline 19.75 & 15.04 & 8.571 & 1.518 & 1.303 & 0.777 & 0.001 & 1.049 & 0.008 & 6.53 & 0.01 & 8.571 & 14.06 & B \\
\hline 19.75 & 39.98 & 6.052 & 4.031 & 1.205 & 0.392 & 0.0003 & 1.077 & 0.003 & 3.29 & 0.003 & 6.052 & 38.66 & B \\
\hline 19.8 & 59.91 & 4.032 & 6.026 & 1.000 & 0.2 & 0.002 & 1.094 & 0.003 & 1.68 & 0.02 & 4.032 & 58.9 & B \\
\hline 19.9 & 71.66 & 2.849 & 7.206 & 0.903 & 0.138 & 0.0003 & 1.105 & 0.002 & 1.16 & 0.003 & 2.849 & 70.84 & B \\
\hline 19.8 & 79.05 & 2.124 & 8.013 & 0.914 & 0.112 & 0.0005 & 1.112 & 0.0007 & 0.94 & 0.004 & 2.124 & 78.3 & B \\
\hline 19.8 & 87.79 & 1.235 & 8.878 & 1.225 & 0.096 & 0.0003 & 1.117 & 0.001 & 0.81 & 0.003 & 1.235 & 87.08 & B \\
\hline 19.8 & 94.77 & 0.526 & 9.538 & 1.414 & 0.104 & 0.006 & 1.12 & 0.054 & 0.87 & 0.05 & 0.526 & 93.95 & B \\
\hline 19.8 & 100 & 0.000 & 10.072 & 1.518 & 0.275 & 0.0005 & 1.123 & 0.004 & 2.31 & 0.005 & 0.000 & 97.69 & B \\
\hline 40.25 & 0 & 10.022 & 0.000 & 2.022 & 1.441 & 0.009 & 1.107 & 0.009 & 12.11 & 0.004 & 10.022 & 0 & B \\
\hline 40.35 & 3.06 & 9.725 & 0.307 & 1.903 & 1.36 & 0.002 & 1.112 & 0.007 & 11.43 & 0.079 & 9.725 & 2.71 & B \\
\hline 40.4 & 15.08 & 8.545 & 1.517 & 1.800 & 1.053 & 0.002 & 1.113 & 0.006 & 8.85 & 0.019 & 8.545 & 13.74 & B \\
\hline 40.4 & 39.88 & 6.034 & 4.003 & 1.611 & 0.543 & 0.001 & 1.09 & 0.019 & 4.56 & 0.016 & 6.034 & 38.07 & B \\
\hline 39.5 & 59.98 & 4.003 & 6.000 & 1.000 & 0.268 & 0.002 & 1.104 & 0.013 & 2.25 & 0.008 & 4.003 & 58.63 & B \\
\hline 40.3 & 71.94 & 2.827 & 7.246 & 1.130 & 0.18 & 0.0001 & 1.111 & 0.012 & 1.51 & 0.019 & 2.827 & 70.85 & B \\
\hline 40.4 & 79.68 & 2.042 & 8.009 & 1.100 & 0.14 & 0.001 & 1.119 & 0.012 & 1.18 & 0.0008 & 2.042 & 78.75 & B \\
\hline 40.5 & 87.74 & 1.239 & 8.867 & 1.302 & 0.117 & 0.001 & 1.122 & 0.011 & 0.98 & 0.01 & 1.239 & 86.88 & B \\
\hline 40.4 & 94.73 & 0.529 & 9.514 & 1.602 & 0.128 & 0.001 & 1.122 & 0.011 & 1.07 & 0.011 & 0.529 & 93.72 & B \\
\hline 39.5 & 100 & 0.000 & 10.024 & 1.911 & 0.353 & 0.001 & 1.134 & 0.01 & 2.97 & 0.01 & 0.000 & 97.03 & B \\
\hline 59.75 & 0 & 10.039 & 0.000 & 2.206 & 1.856 & 0.023 & 1.141 & 0.032 & 15.59 & 0.195 & 10.039 & 0 & B \\
\hline
\end{tabular}




\begin{tabular}{cccccccccccccc}
60.2 & 3.2 & 9.738 & 0.322 & 2.106 & 1.751 & 0.007 & 1.037 & 0.012 & 14.71 & 0.058 & 9.738 & 2.73 & $\mathrm{~B}$ \\
60.25 & 15.17 & 8.528 & 1.525 & 2.027 & 1.366 & 0.011 & 1.146 & 0.028 & 11.48 & 0.094 & 8.528 & 13.43 & $\mathrm{~B}$ \\
60.2 & 40.01 & 6.013 & 4.010 & 1.817 & 0.716 & 0.0005 & 1.108 & 0.044 & 6.01 & 0.004 & 6.013 & 37.6 & $\mathrm{~B}$ \\
57.55 & 60.02 & 4.007 & 6.015 & 1.200 & 0.321 & 0.001 & 1.117 & 0.037 & 2.7 & 0.008 & 4.007 & 58.4 & $\mathrm{~B}$ \\
59.85 & 71.81 & 2.830 & 7.209 & 1.302 & 0.228 & 0.003 & 1.113 & 0.028 & 1.91 & 0.024 & 2.830 & 70.43 & $\mathrm{~B}$ \\
60.05 & 79.83 & 2.046 & 8.097 & 1.321 & 0.171 & 0.0006 & 1.121 & 0.023 & 1.44 & 0.005 & 2.046 & 78.68 & $\mathrm{~B}$ \\
60.3 & 87.95 & 1.208 & 8.818 & 1.501 & 0.139 & 0.0007 & 1.12 & 0.025 & 1.17 & 0.006 & 1.208 & 86.92 & $\mathrm{~B}$ \\
60.2 & 94.84 & 0.518 & 9.508 & 1.807 & 0.146 & 0.0005 & 1.118 & 0.019 & 1.22 & 0.004 & 0.518 & 93.67 & $\mathrm{~B}$ \\
58.3 & 100 & 0.000 & 10.041 & 2.038 & 0.396 & 0.002 & 1.129 & 0.019 & 3.32 & 0.018 & 0.000 & 96.68 & $\mathrm{~B}$ \\
\hline
\end{tabular}

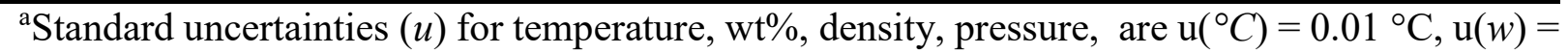
$0.003+0.07, \mathrm{u}\left(S_{T N A}\right)=0.03 \mathrm{~mol} / \mathrm{kg}, \mathrm{u}(p)=1 \mathrm{kPa}$. Standard uncertainties of the densities are estimated as the standard deviations of the mean reported in the table. 
Table 4: Experimental data of the system $\mathrm{NaHCO}_{3}-\mathrm{TEG}-\mathrm{H}_{2} \mathrm{O}$ measured at 5, 20, 40 and $60{ }^{\circ} \mathrm{C}$ and at a pressure $\mathrm{p}=0.1 \mathrm{MPa}^{\mathrm{a}} . t$ is the equilibrium temperature. $\widetilde{w}$ is the salt-free glycol concentration (wt $\%$ ). $\sigma$ is the standard deviation. The results of the RS method was calculated from equations of Fosbøl et al. ${ }^{4,14} \cdot w_{i}$ is the weight percent of component $i$. The solid phase is assumed from a subset of analysis and not every sample was characterized by PXRD.

\begin{tabular}{|c|c|c|c|c|c|c|c|c|c|c|c|c|c|}
\hline \multirow[b]{2}{*}{$\begin{array}{c}\mathrm{t} \\
{ }^{\circ} \mathrm{C} \\
\end{array}$} & \multicolumn{4}{|c|}{ initial } & \multicolumn{4}{|c|}{ equilibrium } & \multicolumn{5}{|c|}{ reverse Schreinemakers } \\
\hline & $\begin{array}{c}\widetilde{w}_{T E G} \\
\%\end{array}$ & $\begin{array}{c}m_{\mathrm{H}_{2} \mathrm{O}} \\
\mathrm{g}\end{array}$ & $\begin{array}{c}m_{T E G} \\
\mathrm{~g}\end{array}$ & $\begin{array}{c}m_{\mathrm{NaHCO}} \\
\mathrm{g}\end{array}$ & $\begin{array}{c}S_{T N a} a \\
\mathrm{~mol} / \mathrm{kg}\end{array}$ & $\begin{array}{c}\sigma_{S_{T N a}} \\
\mathrm{~mol} / \mathrm{kg}\end{array}$ & $\begin{array}{l}\rho_{\text {sat, liq }} \\
\mathrm{g} / \mathrm{cm}^{3}\end{array}$ & $\begin{array}{c}\sigma_{\rho} \\
\mathrm{g} / \mathrm{cm}^{3}\end{array}$ & $\begin{array}{c}w_{\mathrm{NaHCO}_{3}} \\
\%\end{array}$ & $\begin{array}{c}\sigma_{\mathrm{NaHCO}_{3}} \\
\% \\
\end{array}$ & $\begin{array}{c}m_{\mathrm{H}_{2} \mathrm{O}} \\
\mathrm{g}\end{array}$ & $\begin{array}{c}w_{T E G} \\
\%\end{array}$ & $\mathrm{P}$ \\
\hline 5.00 & 76.88 & 4.63 & 15.393 & 0.829 & 0.085 & 0.004 & 1.124 & 0.028 & 0.71 & 0.029 & 4.63 & 76.33 & B \\
\hline 5.00 & 84.94 & 3.014 & 17.002 & 0.844 & 0.068 & 0.0008 & 1.120 & 0.013 & 0.57 & 0.007 & 3.014 & 84.45 & B \\
\hline 4.90 & 91.77 & 1.652 & 18.422 & 0.903 & 0.061 & 0.0005 & 1.091 & 0.025 & 0.51 & 0.004 & 1.652 & 91.30 & B \\
\hline 4.90 & 96.80 & 0.642 & 19.412 & 0.931 & 0.075 & 0.0005 & 1.111 & 0.026 & 0.63 & 0.004 & 0.642 & 96.19 & B \\
\hline 5.20 & 100.0 & 0.000 & 20.018 & 1.058 & 0.055 & 0.005 & 1.105 & 0.036 & 0.46 & 0.044 & 0.000 & 99.54 & B \\
\hline 20.40 & 0.00 & 9.974 & 0.000 & 1.379 & 1.153 & 0.002 & 1.047 & 0.006 & 9.69 & 0.017 & 9.974 & 0.00 & B \\
\hline 20.20 & 2.98 & 9.703 & 0.298 & 1.232 & 1.056 & 0.039 & 1.069 & 0.052 & 8.88 & 0.327 & 9.702 & 2.71 & B \\
\hline 20.20 & 15.28 & 8.504 & 1.533 & 0.976 & 0.794 & 0.001 & 1.052 & 0.003 & 6.67 & 0.012 & 8.504 & 14.26 & B \\
\hline 20.20 & 39.90 & 6.016 & 3.995 & 0.585 & 0.388 & 0.0008 & 1.076 & 0.007 & 3.26 & 0.007 & 6.016 & 38.60 & B \\
\hline 20.20 & 65.08 & 3.5 & 6.522 & 0.349 & 0.154 & 0.002 & 1.098 & 0.013 & 1.29 & 0.021 & 3.5 & 64.24 & B \\
\hline 20.30 & 76.91 & 2.313 & 7.702 & 0.367 & 0.105 & 0.011 & 1.108 & 0.021 & 0.88 & 0.09 & 2.313 & 76.23 & B \\
\hline 20.30 & 85.01 & 1.500 & 8.509 & 0.439 & 0.073 & 0.001 & 1.059 & 0.053 & 0.61 & 0.01 & 1.500 & 84.49 & B \\
\hline 20.20 & 91.77 & 0.829 & 9.246 & 0.341 & 0.070 & 0.001 & 1.082 & 0.055 & 0.59 & 0.027 & 0.829 & 91.23 & B \\
\hline 20.20 & 96.95 & 0.305 & 9.701 & 0.45 & 0.078 & 0.002 & 1.080 & 0.09 & 0.65 & 0.02 & 0.305 & 96.32 & B \\
\hline 20.20 & 100.0 & 0.000 & 10.004 & 0.563 & 0.190 & 0.003 & 1.139 & 0.059 & 1.60 & 0.024 & 0.000 & 98.40 & B \\
\hline 41.10 & 76.92 & 4.635 & 15.445 & 1.1495 & 0.110 & 0.0005 & 1.126 & 0.017 & 0.62 & 0.534 & 4.635 & 76.44 & B \\
\hline 41.30 & 84.80 & 3.052 & 17.020 & 1.1114 & 0.081 & 0.001 & 0.904 & 0.077 & 0.68 & 0.011 & 3.052 & 84.22 & B \\
\hline 41.40 & 91.75 & 1.655 & 18.405 & 1.0542 & 0.069 & 0.003 & 1.121 & 0.003 & 0.58 & 0.025 & 1.655 & 91.22 & B \\
\hline 41.30 & 96.94 & 0.613 & 19.4 & 1.2095 & 0.079 & 0.00003 & 1.129 & 0.035 & 0.66 & 0.0003 & 0.613 & 96.30 & B \\
\hline 40.20 & 100.0 & 0.000 & 20.018 & 1.3155 & 0.210 & 0.002 & 1.168 & 0.018 & 1.77 & 0.021 & 0.000 & 98.23 & B \\
\hline 59.40 & 0.00 & 10.009 & 0.000 & 2.501 & 1.937 & 0.01 & 1.089 & 0.059 & 16.27 & 0.069 & 10.009 & 0.00 & B \\
\hline 59.60 & 3.18 & 9.730 & 0.319 & 2.348 & 1.801 & 0.004 & 1.080 & 0.065 & 15.13 & 0.037 & 9.730 & 2.70 & B \\
\hline 59.80 & 15.13 & 8.501 & 1.515 & 1.614 & 1.413 & 0.01 & 1.065 & 0.064 & 11.87 & 0.087 & 8.501 & 13.33 & B \\
\hline 59.80 & 39.89 & 6.02 & 3.994 & 0.988 & 0.659 & 0.011 & 1.104 & 0.01 & 5.54 & 0.093 & 6.02 & 37.68 & B \\
\hline 59.10 & 64.98 & 3.507 & 6.506 & 0.638 & 0.216 & 0.0009 & 1.111 & 0.008 & 1.81 & 0.007 & 3.507 & 63.80 & B \\
\hline 59.30 & 76.54 & 2.361 & 7.704 & 0.434 & 0.164 & 0.039 & 1.107 & 0.014 & 0.92 & 0.827 & 2.361 & 75.84 & B \\
\hline 59.60 & 84.82 & 1.523 & 8.511 & 0.425 & 0.086 & 0.029 & 1.504 & 0.525 & 0.72 & 0.244 & 1.523 & 84.21 & B \\
\hline 59.70 & 91.96 & 0.808 & 9.248 & 0.554 & 0.074 & 0.007 & 1.132 & 0.006 & 0.62 & 0.056 & 0.808 & 91.39 & B \\
\hline 59.40 & 96.82 & 0.319 & 9.726 & 0.656 & 0.080 & 0.001 & 1.148 & 0.002 & 0.67 & 0.009 & 0.319 & 96.17 & B \\
\hline 58.50 & 100.0 & 0.000 & 9.996 & 0.705 & 0.213 & 0.001 & 1.135 & 0.007 & 1.79 & 0.012 & 0.000 & 98.21 & B \\
\hline
\end{tabular}


aStandard uncertainties $(u)$ for temperature, $\mathrm{wt} \%$, density, pressure, are $\mathrm{u}\left({ }^{\circ} C\right)=0.01{ }^{\circ} \mathrm{C}, \mathrm{u}(w)=$ $0.003+0.07, \mathrm{u}\left(S_{T N A}\right)=0.03 \mathrm{~mol} / \mathrm{kg}, \mathrm{u}(p)=1 \mathrm{kPa}$. Standard uncertainties of the densities are estimated as the standard deviations of the mean reported in the table.

Table 5. Experimental data of the system $\mathrm{NaHCO}_{3}-\mathrm{TeEG}-\mathrm{H}_{2} \mathrm{O}$ measured at 5, 20, 40 and $60{ }^{\circ} \mathrm{C}$ and at a pressure $\mathrm{p}=0.1 \mathrm{MPa}^{\mathrm{a}} . t$ is the equilibrium temperature. $\widetilde{w}$ is the salt-free glycol concentration (wt\%). $\sigma$ is the standard deviation. The results of the RS method was calculated from equations of Fosbøl et al. ${ }^{4,14}$. $w_{i}$ is the weight percent of component $i$. The solid phase is assumed from a subset of analysis and not every sample was characterized by PXRD.

\begin{tabular}{|c|c|c|c|c|c|c|c|c|c|c|c|c|c|}
\hline \multirow[b]{2}{*}{$\begin{array}{c}\mathrm{t} \\
{ }^{\circ} \mathrm{C} \\
\end{array}$} & \multicolumn{4}{|c|}{ initial } & \multicolumn{4}{|c|}{ equilibrium } & \multicolumn{5}{|c|}{ reverse Schreinemakers } \\
\hline & $\begin{array}{c}\widetilde{w}_{T e E G} \\
\%\end{array}$ & $\begin{array}{c}m_{\mathrm{H}_{2} \mathrm{O}} \\
\mathrm{g}\end{array}$ & $\begin{array}{c}m_{T e E G} \\
\mathrm{~g}\end{array}$ & $\begin{array}{c}m_{\mathrm{NaHCO}_{3}} \\
\mathrm{~g}\end{array}$ & $\begin{array}{c}S_{T N a} \\
\mathrm{~mol} / \mathrm{kg}\end{array}$ & $\begin{array}{c}\sigma_{S_{T N a}} \\
\mathrm{~mol} / \mathrm{kg}\end{array}$ & $\begin{array}{l}\rho_{\text {sat, liq }} \\
\mathrm{g} / \mathrm{cm}^{3}\end{array}$ & $\begin{array}{c}\sigma_{\rho} \\
\mathrm{g} / \mathrm{cm}^{3}\end{array}$ & $\begin{array}{c}w_{\mathrm{NaHCO}_{3}} \\
\%\end{array}$ & $\begin{array}{c}\sigma_{\mathrm{NaHCO}_{3}} \\
\%\end{array}$ & $\begin{array}{c}m_{\mathrm{H}_{2} \mathrm{O}} \\
\mathrm{g}\end{array}$ & $\begin{array}{c}w_{T e E G} \\
\%\end{array}$ & $\mathrm{P}$ \\
\hline 5.40 & 0.00 & 20.010 & 0.000 & 2.479 & 0.842 & 0.004 & 1.052 & 0.012 & 7.08 & 0.034 & 20.010 & 0.00 & B \\
\hline 5.40 & 3.00 & 19.422 & 0.6 & 2.238 & 0.780 & 0.008 & 1.050 & 0.013 & 6.55 & 0.067 & 19.422 & 2.80 & B \\
\hline 5.30 & 14.96 & 17.035 & 2.998 & 2.062 & 0.584 & 0.005 & 1.048 & 0.006 & 4.90 & 0.041 & 17.035 & 14.23 & B \\
\hline 5.30 & 39.88 & 12.058 & 7.999 & 1.264 & 0.263 & 0.003 & 1.081 & 0.011 & 2.21 & 0.026 & 12.058 & 39.00 & B \\
\hline 5.70 & 66.81 & 6.658 & 13.399 & 0.901 & 0.094 & 0.005 & 1.130 & 0.018 & 0.79 & 0.045 & 6.658 & 66.28 & B \\
\hline 4.90 & 78.49 & 4.337 & 15.829 & 0.872 & 0.063 & 0.001 & 1.116 & 0.016 & 0.53 & 0.011 & 4.337 & 78.08 & B \\
\hline 4.90 & 87.82 & 2.441 & 17.604 & 0.899 & 0.041 & 0.001 & 1.107 & 0.053 & 0.35 & 0.009 & 2.441 & 87.52 & B \\
\hline 4.80 & 93.90 & 1.222 & 18.822 & 0.870 & 0.037 & 0.0004 & 1.172 & 0.1 & 0.31 & 0.003 & 1.222 & 93.62 & B \\
\hline 4.80 & 97.42 & 0.516 & 19.508 & 1.124 & 0.034 & 0.0004 & 1.157 & 0.109 & 0.28 & 0.0004 & 0.516 & 97.15 & B \\
\hline 5.20 & 100.0 & 0.000 & 20.031 & 1.314 & 0.037 & 0.002 & 1.148 & 0.035 & 0.31 & 0.02 & 0.000 & 99.69 & B \\
\hline 20.00 & 87.80 & 2.448 & 17.623 & 0.836 & 0.044 & 0.008 & 1.131 & 0.026 & 0.37 & 0.066 & 2.448 & 87.48 & B \\
\hline 20.00 & 93.82 & 1.239 & 18.801 & 0.847 & 0.036 & 0.001 & 1.165 & 0.027 & 0.30 & 0.012 & 1.239 & 93.53 & B \\
\hline 20.00 & 97.35 & 0.530 & 19.500 & 1.114 & 0.052 & 0.0005 & 1.142 & 0.01 & 0.43 & 0.005 & 0.530 & 96.93 & B \\
\hline 20.00 & 100.0 & 0.000 & 20.056 & 1.275 & 0.136 & 0.013 & 1.097 & 0.033 & 1.14 & 0.108 & 0.000 & 98.86 & B \\
\hline 20.10 & 3.00 & 19.418 & 0.602 & 3.533 & 1.021 & 0.001 & 1.079 & 0.015 & 8.58 & 0.01 & 19.418 & 2.75 & B \\
\hline 39.60 & 0.00 & 9.994 & 0.000 & 1.723 & 1.492 & 0.0009 & 1.085 & 0.034 & 12.53 & 0.007 & 9.994 & 0.00 & B \\
\hline 40.00 & 3.19 & 9.712 & 0.32 & 1.678 & 1.249 & 0.054 & 1.114 & 0.027 & 10.49 & 0.451 & 9.712 & 2.85 & B \\
\hline 39.90 & 15.03 & 8.548 & 1.512 & 1.239 & 0.973 & 0.008 & 1.092 & 0.02 & 8.17 & 0.066 & 8.548 & 13.80 & B \\
\hline 39.90 & 39.94 & 6.021 & 4.004 & 0.779 & 0.437 & 0.033 & 1.123 & 0.078 & 3.67 & 0.274 & 6.021 & 38.48 & B \\
\hline 39.20 & 66.73 & 3.348 & 6.716 & 0.588 & 0.134 & 0.005 & 1.121 & 0.007 & 1.13 & 0.039 & 3.348 & 65.98 & B \\
\hline 39.60 & 78.65 & 2.145 & 7.902 & 0.415 & 0.083 & 0.004 & 1.112 & 0.021 & 0.70 & 0.033 & 2.145 & 78.11 & B \\
\hline 40.00 & 87.86 & 1.219 & 8.820 & 0.43 & 0.049 & 0.0002 & 1.120 & 0.01 & 0.41 & 0.001 & 1.219 & 87.50 & B \\
\hline 40.00 & 93.81 & 0.621 & 9.41 & 0.418 & 0.039 & 0.001 & 1.136 & 0.008 & 0.33 & 0.012 & 0.621 & 93.50 & B \\
\hline 39.90 & 97.09 & 0.293 & 9.747 & 0.662 & 0.039 & 0.0004 & 1.129 & 0.01 & 0.33 & 0.003 & 0.293 & 96.77 & B \\
\hline 39.20 & 100.00 & 0.000 & 10.038 & 0.695 & 0.067 & 0.004 & 1.105 & 0.063 & 0.56 & 0.037 & 0.000 & 99.44 & B \\
\hline 59.50 & 0.00 & 10.033 & 0.000 & 2.316 & 1.827 & 0.007 & 1.112 & 0.034 & 15.35 & 0.055 & 10.033 & 0.00 & B \\
\hline 59.50 & 3.15 & 9.734 & 0.317 & 2.172 & 1.513 & 0.001 & 1.109 & 0.037 & 12.71 & 0.011 & 9.734 & 2.75 & B \\
\hline 60.00 & 14.90 & 8.528 & 1.494 & 1.737 & 1.134 & 0.007 & 1.094 & 0.031 & 9.52 & 0.06 & 8.528 & 13.48 & B \\
\hline
\end{tabular}




\begin{tabular}{cccccccccccccc}
60.60 & 40.11 & 6.026 & 4.036 & 1.062 & 0.524 & 0.001 & 1.102 & 0.032 & 4.40 & 0.012 & 6.026 & 38.34 & $\mathrm{~B}$ \\
58.20 & 66.69 & 3.345 & 6.698 & 0.644 & 0.143 & 0.001 & 1.096 & 0.024 & 1.20 & 0.009 & 3.345 & 65.89 & $\mathrm{~B}$ \\
60.60 & 78.65 & 2.141 & 7.899 & 0.569 & 0.099 & 0.016 & 1.112 & 0.003 & 0.76 & 0.007 & $\mathrm{~N} / \mathrm{a}$ & 78.06 & $\mathrm{~B}$ \\
60.90 & 87.49 & 1.256 & 8.791 & 0.531 & 0.059 & 0.003 & 1.118 & 0.021 & 0.50 & 0.028 & 1.256 & 87.06 & $\mathrm{~B}$ \\
61.50 & 93.52 & 0.651 & 9.398 & 0.586 & 0.039 & 0.002 & 1.130 & 0.021 & 0.33 & 0.017 & 0.651 & 93.21 & $\mathrm{~B}$ \\
61.40 & 97.27 & 0.275 & 9.773 & 0.756 & 0.044 & 0.003 & 1.076 & 0.082 & 0.37 & 0.025 & 0.275 & 96.91 & $\mathrm{~B}$ \\
60.40 & 100.00 & 0.000 & 10.021 & 0.89 & 0.148 & 0.007 & 0.591 & 0.1 & 1.24 & 0.056 & 0.000 & 98.76 & $\mathrm{~B}$ \\
\hline
\end{tabular}

${ }^{\mathrm{a}}$ Standard uncertainties $(u)$ for temperature, $\mathrm{wt} \%$, density, pressure, are $\mathrm{u}\left({ }^{\circ} \mathrm{C}\right)=0.01{ }^{\circ} \mathrm{C}, \mathrm{u}(w)=$ $0.003+0.07, \mathrm{u}\left(S_{T N A}\right)=0.03 \mathrm{~mol} / \mathrm{kg}, \mathrm{u}(p)=1 \mathrm{kPa}$. Standard uncertainties of the densities are estimated as the standard deviations of the mean reported in the table.

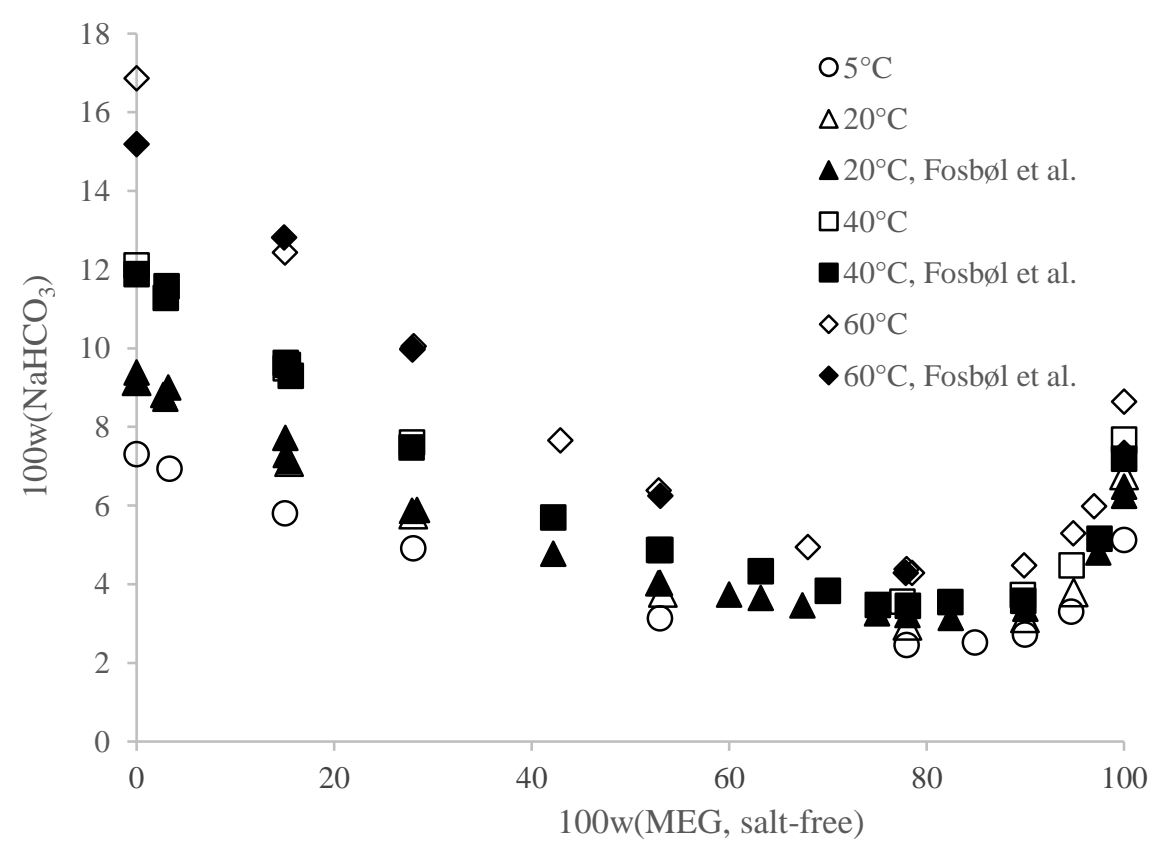

Figure 1. Solubility of $\mathrm{NaHCO}_{3}$ in the aqueous MEG system measured in this work at atmospheric conditions in the temperature range 5 to $60^{\circ} \mathrm{C}$. 


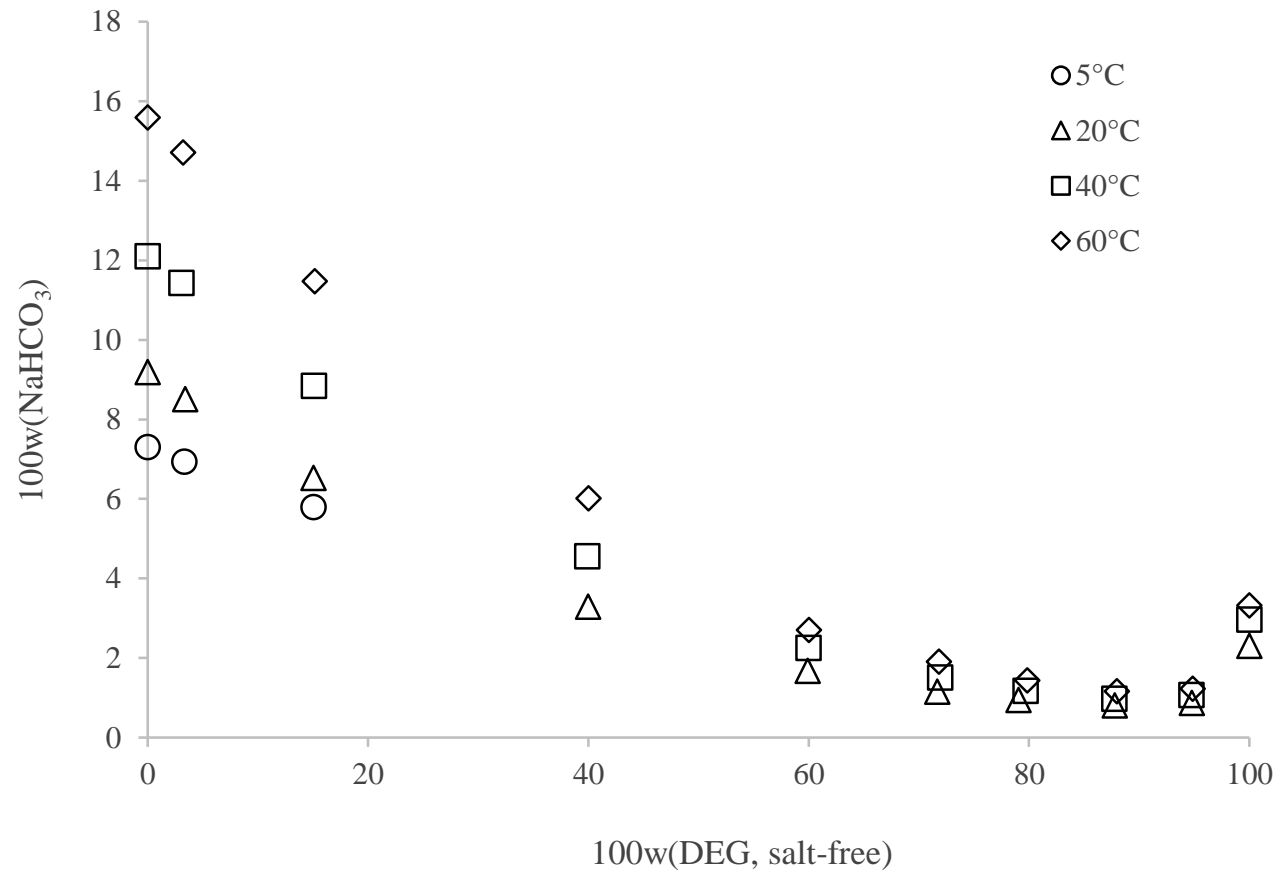

Figure 2. Solubility of $\mathrm{NaHCO}_{3}$ in the aqueous DEG system measured in this work at atmospheric conditions in the temperature range 5 to $60^{\circ} \mathrm{C}$.

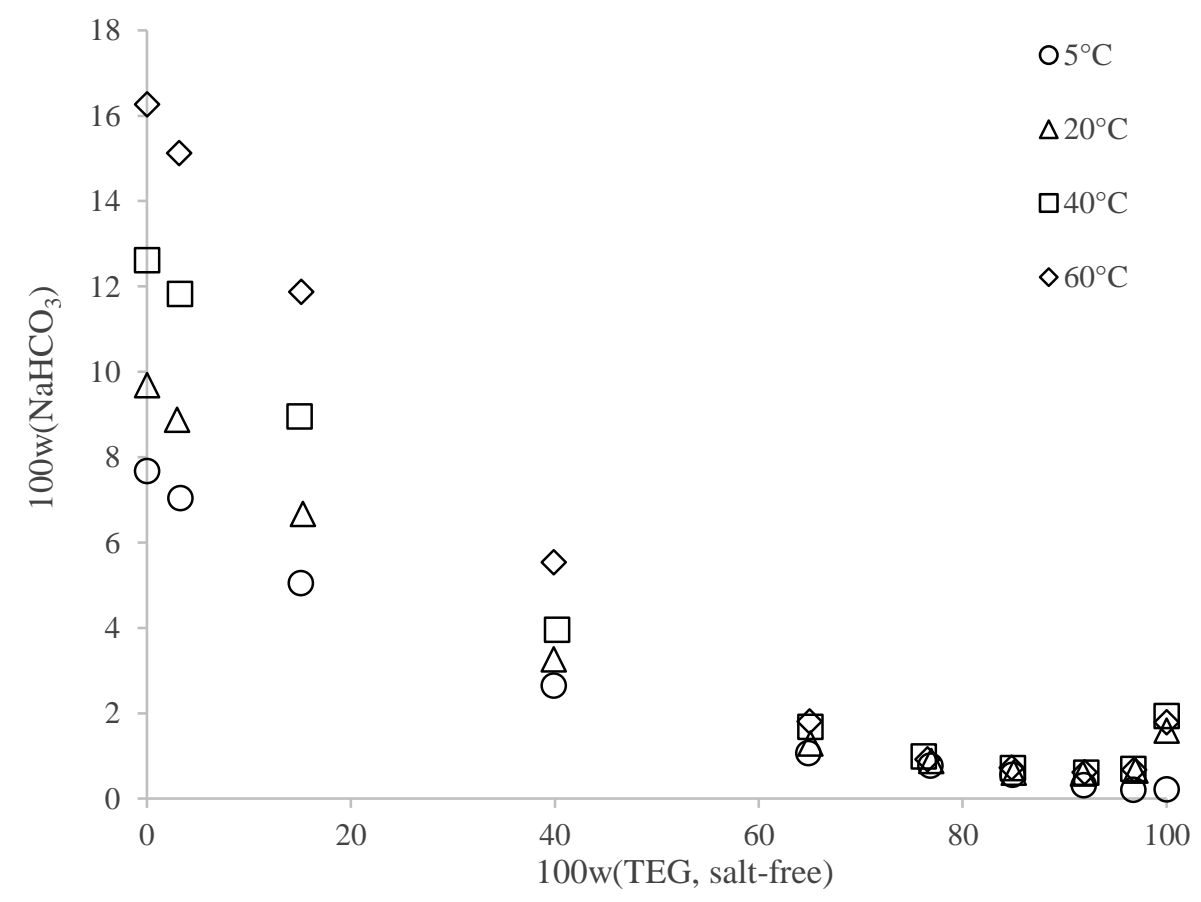


Figure 3. Solubility of $\mathrm{NaHCO}_{3}$ in the aqueous TEG system measured in this work at atmospheric conditions in the temperature range 5 to $60{ }^{\circ} \mathrm{C}$.

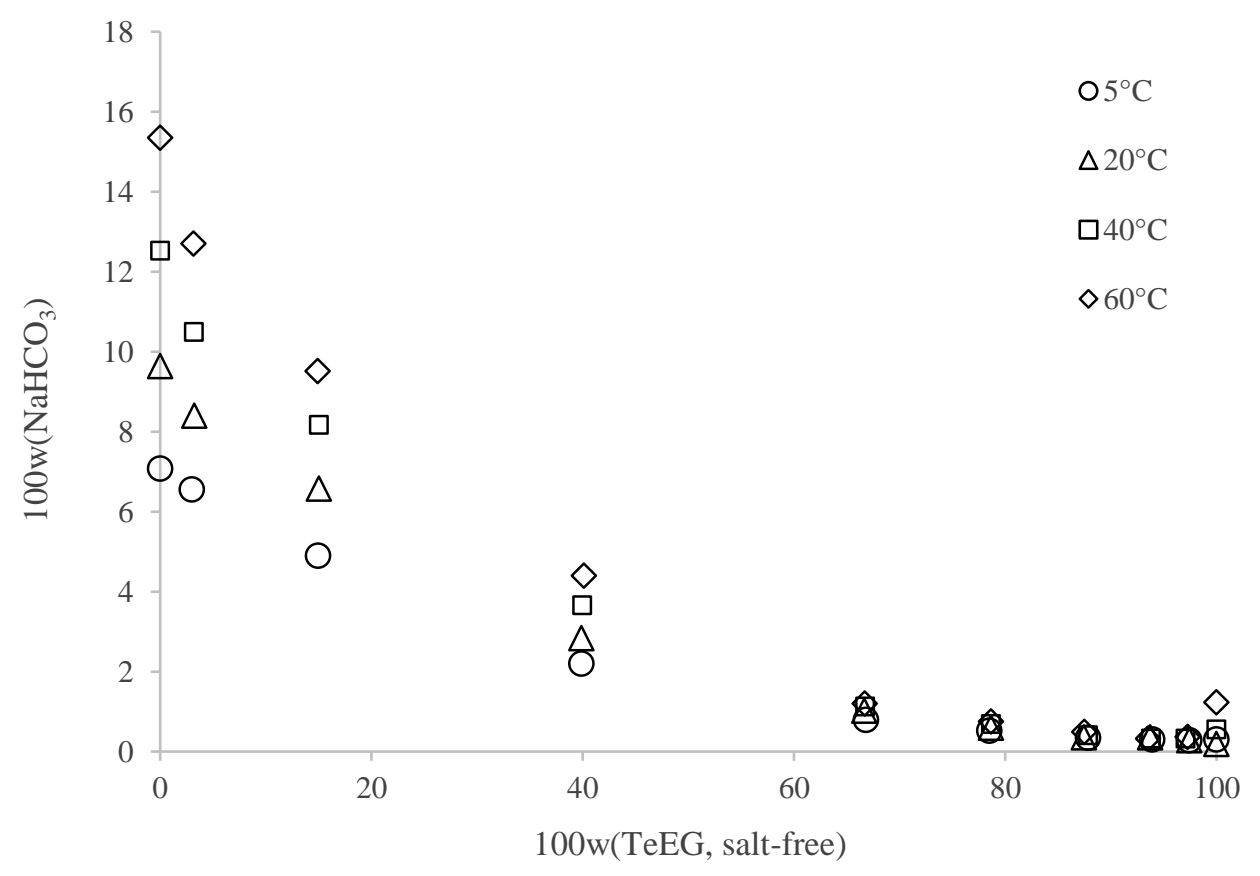

Figure 4. Solubility of $\mathrm{NaHCO}_{3}$ in the aqueous TeEG system measured in this work at atmospheric conditions in the temperature range 5 to $60^{\circ} \mathrm{C}$.

Figure 5 illustrates the solubility of $\mathrm{NaHCO}_{3}$ in pure water and at constant glycol (salt-free) concentrations of ${ }_{\approx} 15$ and ${ }_{\approx} 95 \mathrm{wt} \%$ as a function of temperature. Our experimental results for the solubility of $\mathrm{NaHCO}_{3}$ in water are validated towards previously published data ${ }^{16-31}$, Figure 5 . The experimental data are in line with the previously published data up to $40{ }^{\circ} \mathrm{C}$. At $60^{\circ} \mathrm{C}$ our measured data tend to deviate with an upward trend compared with previously published data. The amount of published data available at this temperature is non-existent.

The $\mathrm{NaHCO}_{3}$ solubility is highest in pure water and decreasing with increased amount of glycol. The solubility of $\mathrm{NaHCO}_{3}$ is also decreasing with higher glycol molecular weight, and linearly increasing with temperature. At high glycol concentration, the temperature effect on the salt 
solubility is scarcely seen except in the MEG-water system. The explanation may be increased $\mathrm{CO}_{2}$ concentration at high glycol concentration or the formation of glycol-bicarbonate complexes.

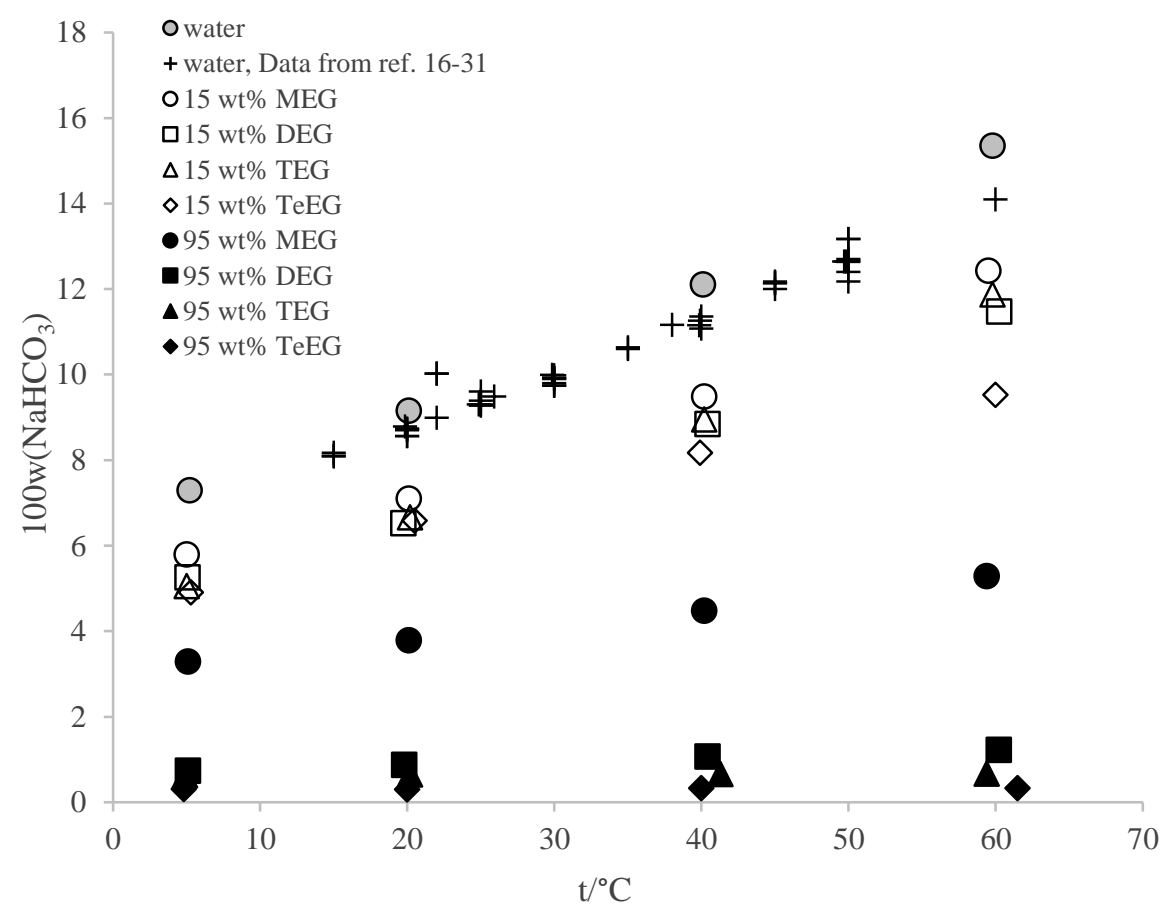

Figure 5. $\mathrm{NaHCO}_{3}$ solubility as a function of temperature. Previously published data ${ }^{16-31}$ for $\mathrm{NaHCO}_{3}$-water are plotted for comparison.

The $\mathrm{NaHCO}_{3}$ solubility at $20{ }^{\circ} \mathrm{C}$ in four aqueous glycol systems are plotted in Figure 6 . The $\mathrm{NaHCO}_{3}$ solubility (wt\%) is given on logarithmic scale and the glycol (salt-free) concentration is in weight percent on the horizontal axis.

It is evident that the solubility increases significantly from its value in aqueous MEG, through higher molecular weights of glycols. The minima in Figure 6 move toward higher glycol concentration increasing the molecular weight of the glycol homologues. In all glycol systems, the solubility is declining at low glycol concentrations compared to the value in pure water. After a minimum solubility, the solubility increases by adding more glycol. 
An interesting phenomenon is observed by plotting the $\mathrm{NaHCO}_{3}$ logarithmic solubility as function of glycol mole fraction. Comparing to the figure of weight percent of $\mathrm{NaHCO}_{3}($ Figure 7 , Figure 8, Figure 9, and Figure 10) it graphically gives a rather different representation of the solubility curve. The curves all have a parabolic trend and the influence of the glycol molecular mass is even more distinct. The logarithmic scale indicates that the temperature has insignificant impact on the $\mathrm{NaHCO}_{3}$ solubility but this is not quite the case as seen in Figure 1, Figure 2, Figure 3, and Figure 4. An outlier related to the increased viscosity for $100 \mathrm{wt} \%$ TEG is observed in Figure 7.

On a mole fraction scale, there is a clear and visible minimum at $x$ (glycol, salt-free $)=0.5$. The minimum remains quite constant at $50 \mathrm{~mol} \%( \pm 5 \%)$ in all systems at all temperatures. This minimum could be explained by a 1:1 $\mathrm{NaHCO}_{3}$ solvation by either water or glycol. Water and $\mathrm{NaHCO}_{3}$ apparently have a tendency to form one type of $\mathrm{NaHCO}_{3}$ solvation while glycol-NaHCO 3 forms another. The affinity for $\mathrm{NaHCO}_{3}$ appears to be equally shared by water and glycol. Therefore we see this minimum at $x$ (glycol, salt-free $)=0.5$ where $\mathrm{NaHCO}_{3}$ is neither bound to water nor to glycol, but in a transition from one molecular relation to the other. The two solvent molecules tend to interact with each other, rather than with the sodium and the bicarbonate ions. This results in salting out of $\mathrm{NaHCO}_{3}$ because the salt is unable to remain solubilized in the mixture.

The increased $\mathrm{NaHCO}_{3}$ solubility at high glycol concentration can also be explained in a different way. It may be influenced as explained by Gärtner et al. ${ }^{12}$ by soluble complexes of glycol and $\mathrm{HCO}_{3}^{-}$or un-dissociated $\mathrm{NaHCO}_{3}$ where water replaces $\mathrm{HCO}_{3}^{-}$due to the capability of water to form hydrogen bonding. At higher content of water in the mixtures the solubility of $\mathrm{HCO}_{3}^{-}$ increases as cavity forms in the solvent making space for $\mathrm{HCO}_{3}^{-}$. The solvating effect at high 
glycol concentration indicates that glycol and $\mathrm{HCO}_{3}^{-}$form complexes. Gärtner et al. ${ }^{32}$ explain that the solvation mechanism in almost pure glycol might be due to glycol forming hydrogen bridges with $\mathrm{HCO}_{3}^{-}$, increasing the solubility of bicarbonate ions.

Solubilisation of salts and ions are often strongly related to the static permittivity of the solvent. The tendency of the solvent to shield or transfer the coulombic forces from $\mathrm{Na}^{+}$or $\mathrm{HCO}_{3}{ }^{-}$to one of the solvent molecules is given by this property. Therefore the solubility of molecules can have a strong relation to static permittivity. The clear minimum in solubility observed is not due to changes in static permittivity. Previous work ${ }^{33}$ show that static permittivity for the mixed waterglycol solvent has no minimum in the static permittivity and the solubility minimum must therefore originate from other types of phenomena. This could be hydrogen bonds between $\mathrm{NaHCO}_{3}$ and either of the solvent molecules. The minimum observed in this system is also very different from similar mixed solvent salt systems. The minimum is not observed in e.g. NaCl-MEG- $\mathrm{H}_{2} \mathrm{O}^{34}$ or $\mathrm{Na}_{2} \mathrm{SO}_{4}-\mathrm{MEG}-\mathrm{H}_{2} \mathrm{O}^{35}$, where the bond must be different apparently. 


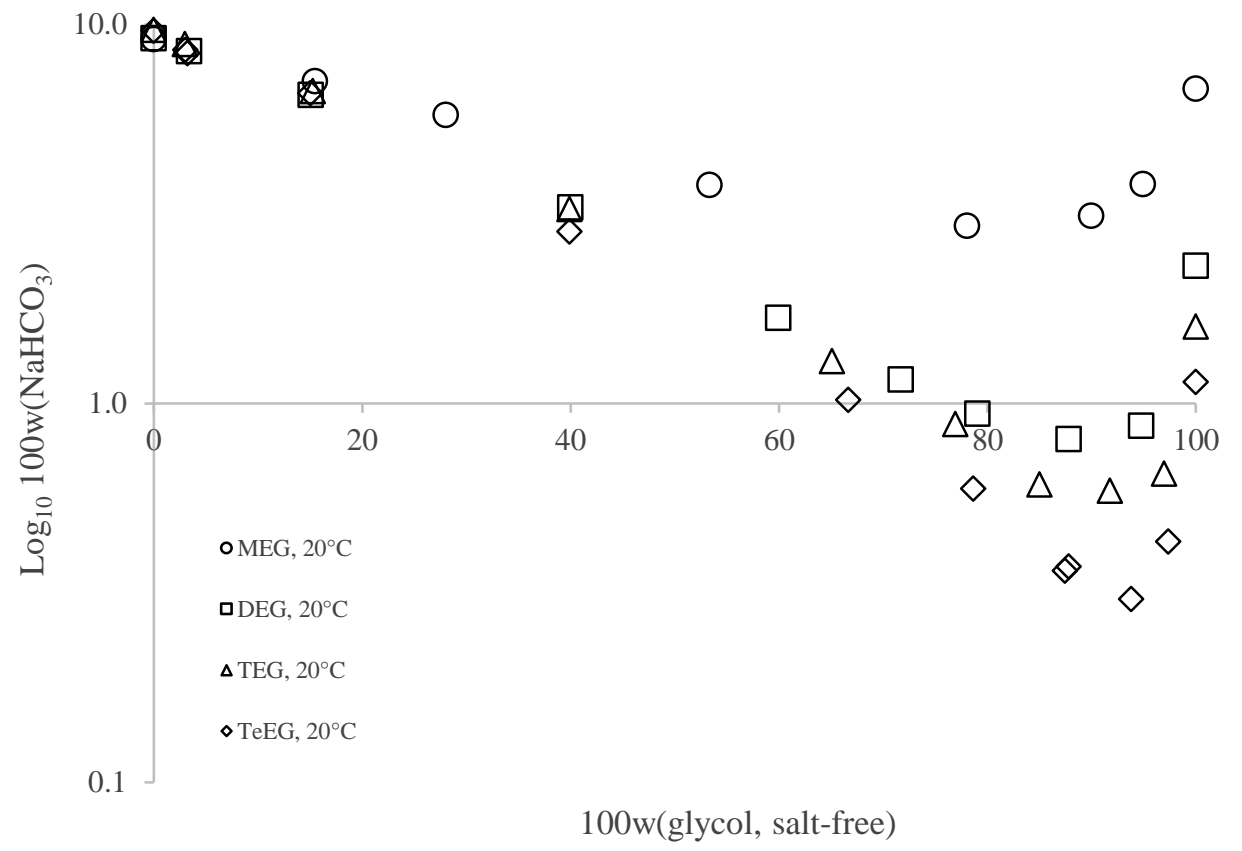

Figure 6. Solubility of $\mathrm{NaHCO}_{3}(\mathrm{wt} \%)$ in glycol-water mixtures (wt\%) at $20{ }^{\circ} \mathrm{C}$.

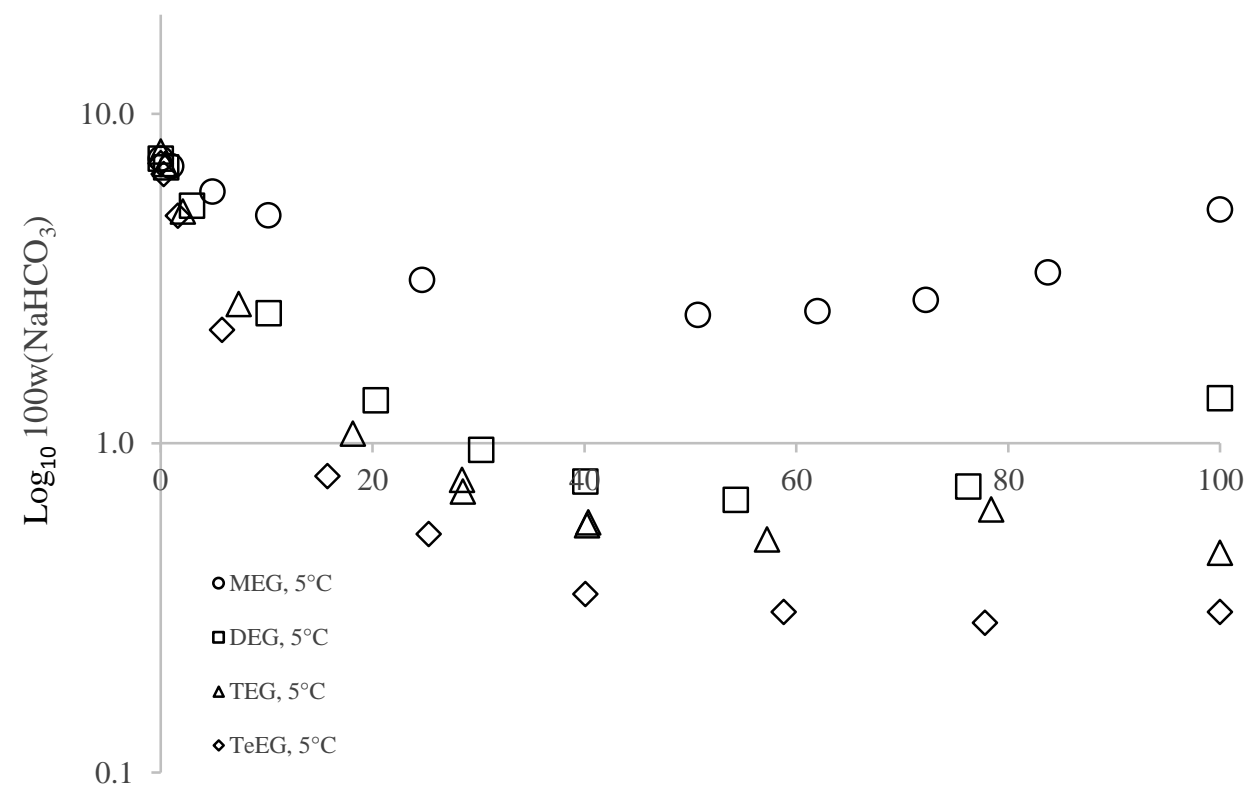

100x(glycol, salt-free)

Figure 7. Solubility of $\mathrm{NaHCO}_{3}(\mathrm{wt} \%)$ in glycol-water mixtures $(\mathrm{mole} \%)$ at $5{ }^{\circ} \mathrm{C}$. 


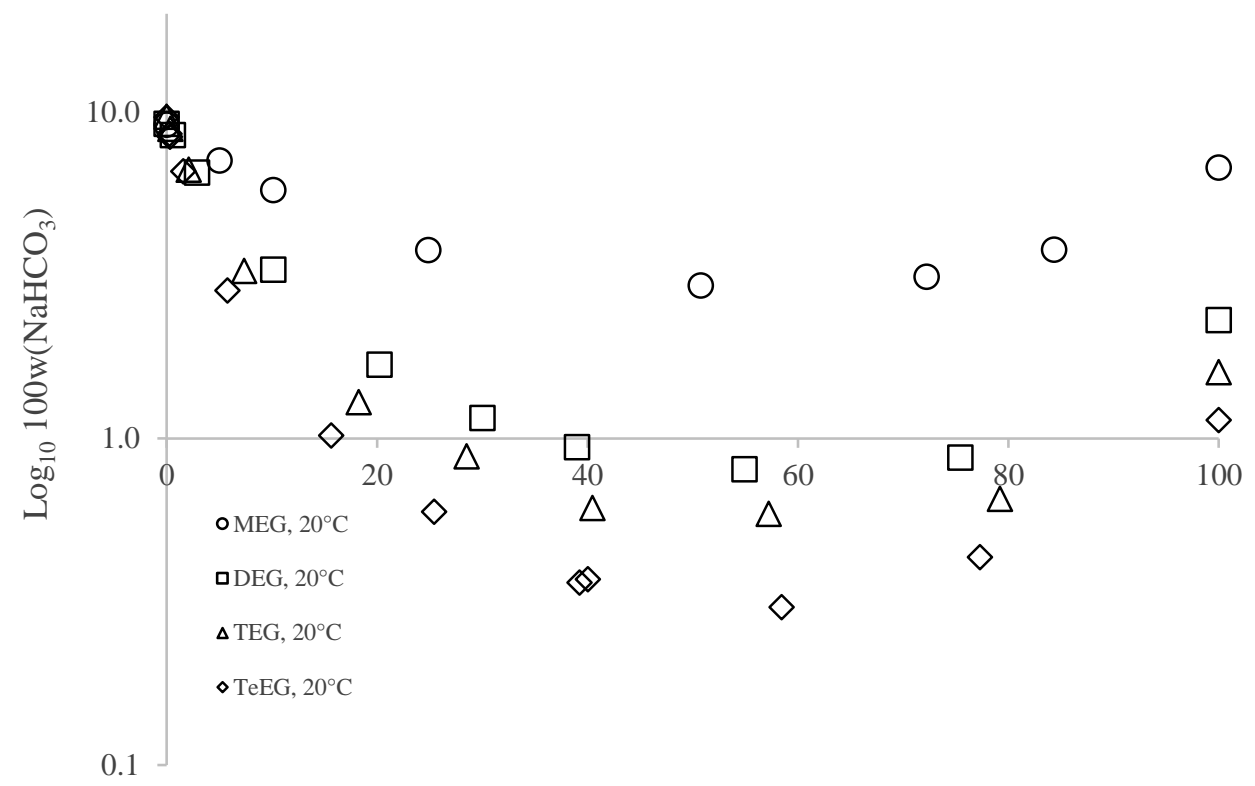

100x(glycol, salt-free)

Figure 8. Solubility of $\mathrm{NaHCO}_{3}(\mathrm{wt} \%)$ in glycol-water mixtures $(\mathrm{mole} \%)$ at $20{ }^{\circ} \mathrm{C}$.

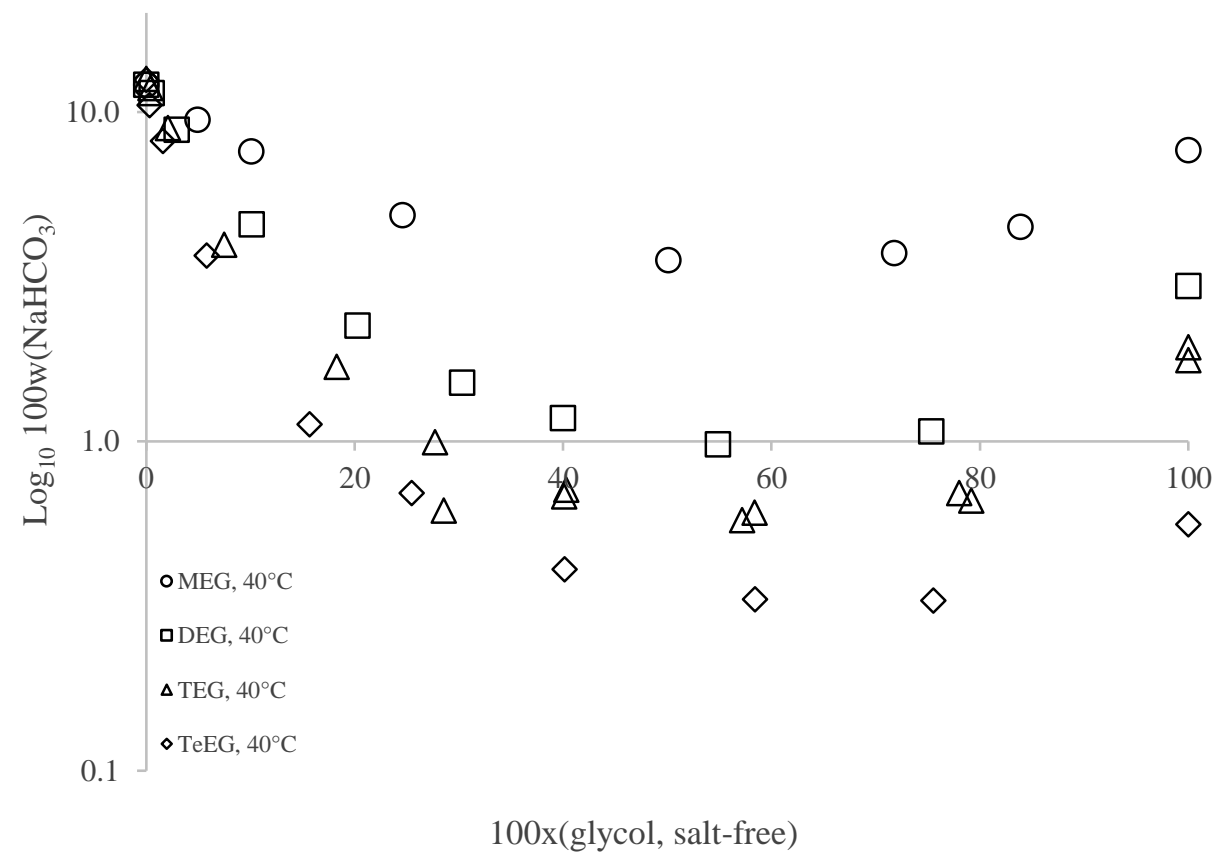

Figure 9. Solubility of $\mathrm{NaHCO}_{3}(\mathrm{wt} \%)$ in glycol-water mixtures $($ mole $\%)$ at $40{ }^{\circ} \mathrm{C}$. 


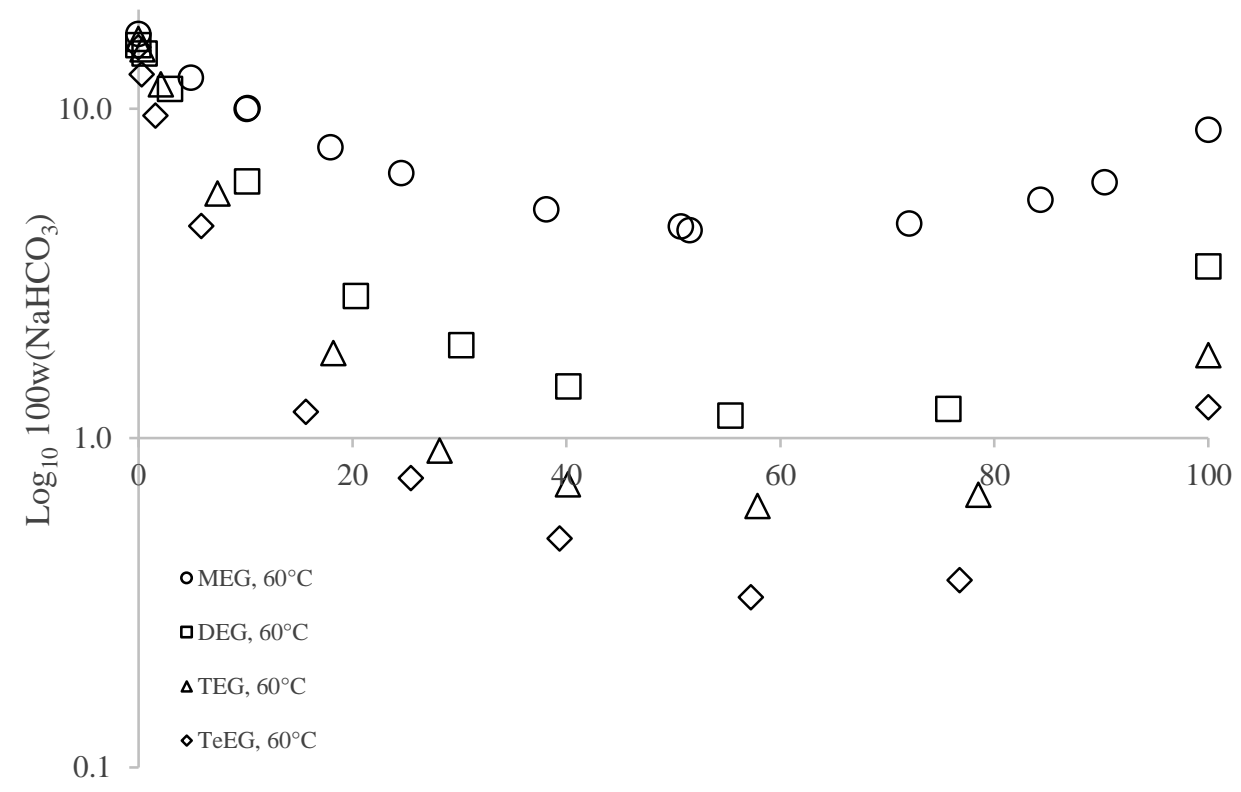

100x(glycol, salt-free)

Figure 10. Solubility of $\mathrm{NaHCO}_{3}(\mathrm{wt} \%)$ in glycol-water mixtures (mole \%) at $60{ }^{\circ} \mathrm{C}$.

The reproducibility of the density measurements are listed in Table 2, Table 3, Table 4, and Table 5. The density was measured by taking 100 to $1000 \mu \mathrm{L}$ of a sample and weighing it. This method is giving standard deviations up to $2 \%$, which is relatively high. The reasons for the high deviations were explained by Fosbøl et al. ${ }^{11}$ to be caused by air intake by the micropipette or too small sample sizes.

Figure 11, Figure 12, Figure 13, and Figure 14 depict the measured densities at different isotherms of $\mathrm{NaHCO}_{3}-\mathrm{MEG}-\mathrm{H}_{2} \mathrm{O}, \mathrm{NaHCO}_{3}-\mathrm{DEG}-\mathrm{H}_{2} \mathrm{O}, \mathrm{NaHCO}_{3}-\mathrm{TEG}-\mathrm{H}_{2} \mathrm{O}$, and $\mathrm{NaHCO}_{3}$ TeEG- $\mathrm{H}_{2} \mathrm{O}$ mixtures. The densities for all systems increase with increasing temperature. There seems to be a slight tendency in lower density from MEG to TeEG.

The results of the density measured for saturated $\mathrm{NaHCO}_{3}-\mathrm{MEG}-\mathrm{H}_{2} \mathrm{O}$ mixtures, Figure 11 , were compared to the data by Fosbøl et al. ${ }^{11}$. The density data seem to be more temperature dependent 
at low MEG concentrations. From $60 \mathrm{wt} \%$ to $100 \mathrm{wt} \%$ MEG the density does not vary much with temperature. This was also observed in the solid-liquid equilibrium (SLE) diagram, Figure 1.

The density of measured $\mathrm{NaHCO}_{3}-\mathrm{DEG}-\mathrm{H}_{2} \mathrm{O}$ mixtures, Figure 12, has tendencies similar to those of $\mathrm{NaHCO}_{3}-\mathrm{MEG}-\mathrm{H}_{2} \mathrm{O}$ mixtures. At low DEG concentrations, the density is increasing linearly for the isotherms 5 and $20^{\circ} \mathrm{C}$. At 40 and $60{ }^{\circ} \mathrm{C}$, the density is decreasing. The behaviour might be due to the experimental uncertainty. At high glycol concentrations, the density is following the solubility trend.

For the system, $\mathrm{NaHCO}_{3}-\mathrm{TEG}-\mathrm{H}_{2} \mathrm{O}$ the temperature only has little influence on the density. The density is weakly increasing up to $75 \mathrm{wt} \%$ TEG and around $85 \mathrm{wt} \%$ TEG the density may have a minimum similar to the one observed in the SLE phase diagram Figure 13. Two outliers are shown at 40 and $60^{\circ} \mathrm{C}$ at a TEG concentration of approximately $85 \mathrm{wt} \%$. The outliers are caused by the limitation of the density method. Figure 14 illustrates the density of $\mathrm{NaHCO}_{3}-\mathrm{TeEG}-\mathrm{H}_{2} \mathrm{O}$. The trend of this system is deviating from the other glycol systems, as the temperature seems to have an insignificant impact on the density. The behaviour may be caused by density being difficult to measure due to high viscosity. 


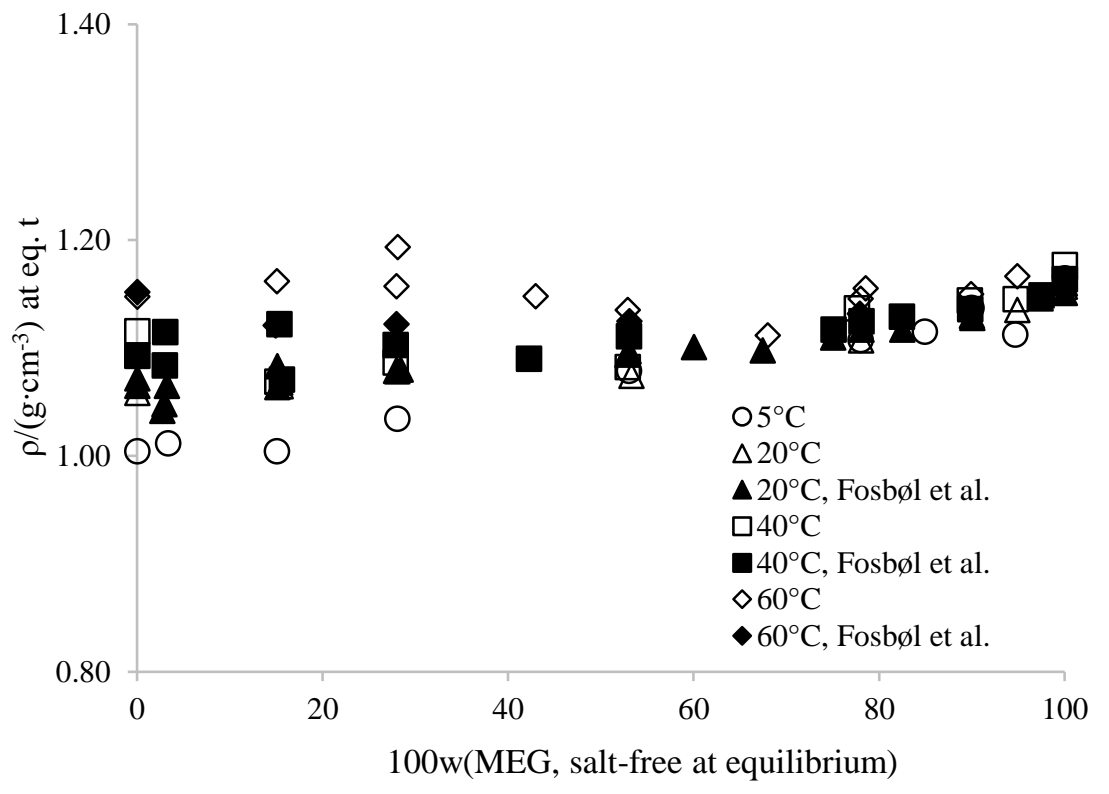

Figure 11: Density of saturated $\mathrm{NaHCO}_{3}-\mathrm{MEG}-\mathrm{H}_{2} \mathrm{O}$ solutions measured at selected isotherms at atmospheric pressure.

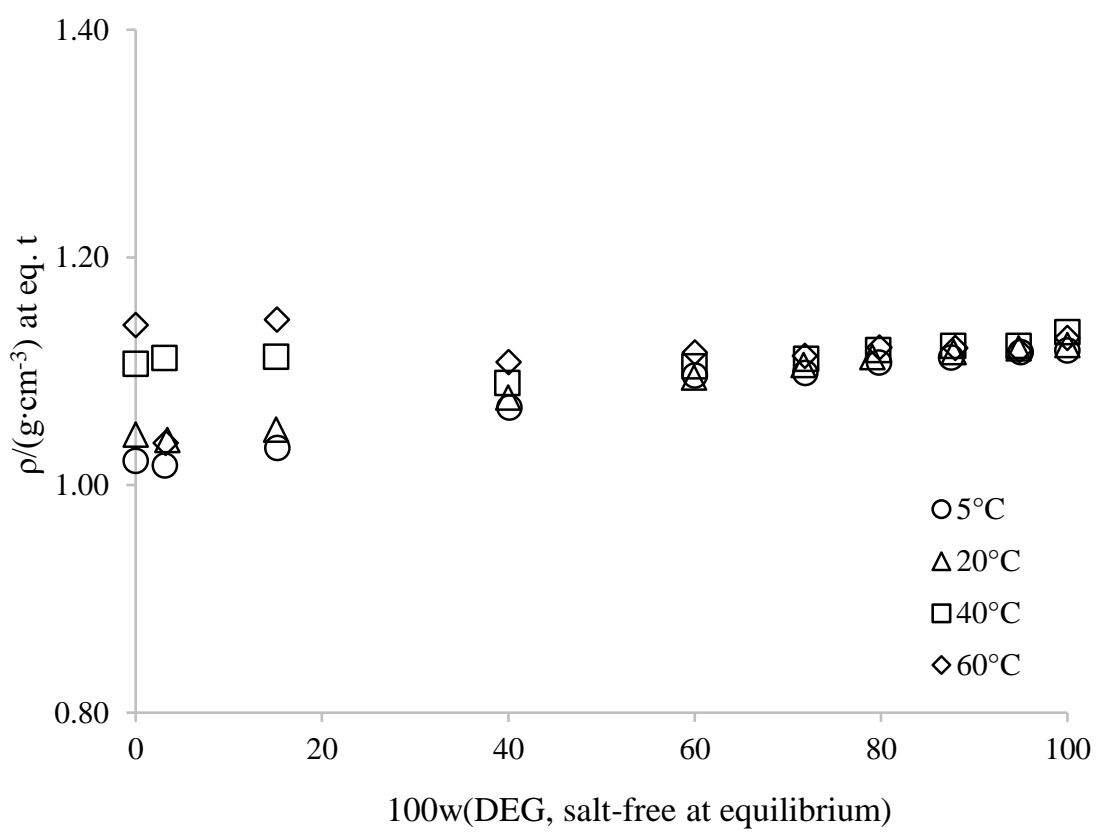

Figure 12: Density of saturated $\mathrm{NaHCO}_{3}-\mathrm{DEG}-\mathrm{H}_{2} \mathrm{O}$ solutions measured at selected isotherms at atmospheric pressure. 


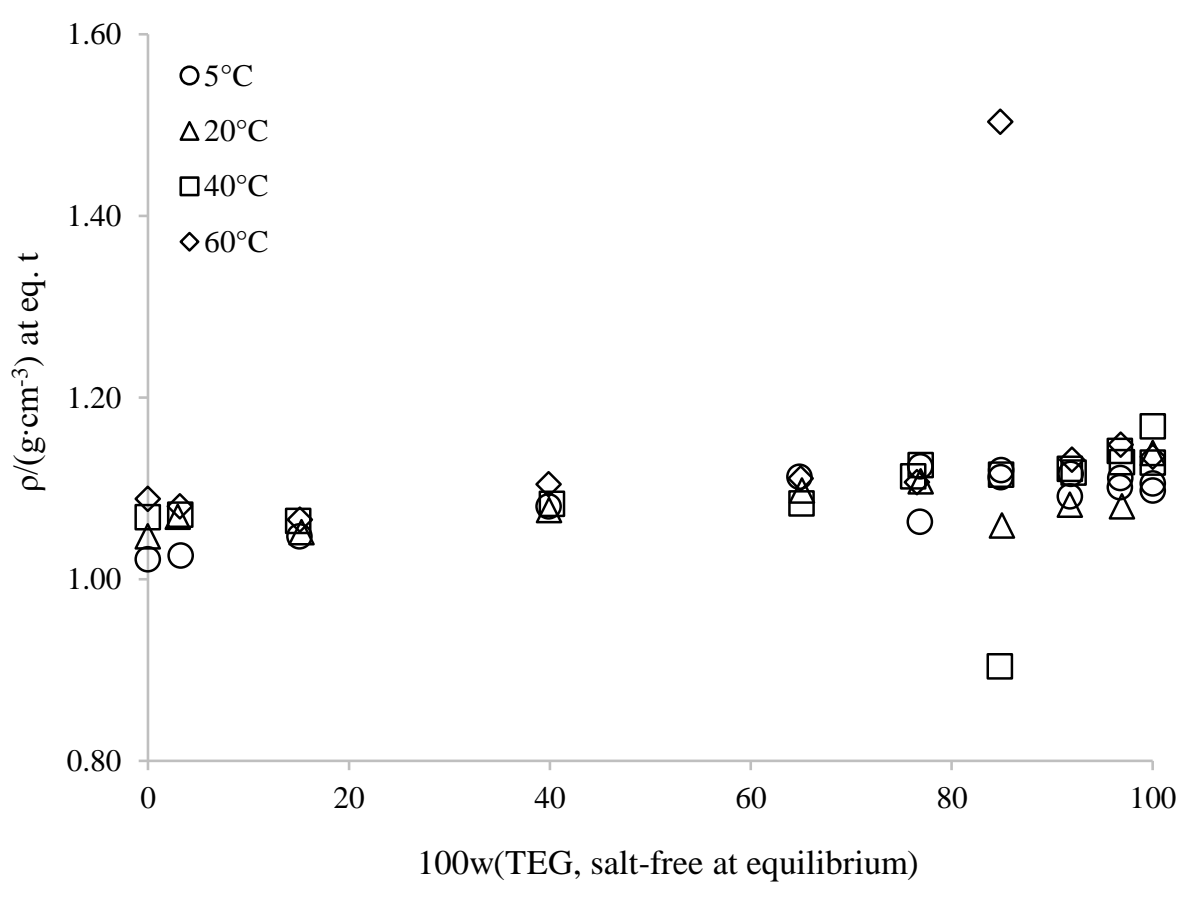

Figure 13: Density of saturated $\mathrm{NaHCO}_{3}-\mathrm{TEG}-\mathrm{H}_{2} \mathrm{O}$ solutions measured at selected isotherms at atmospheric pressure.

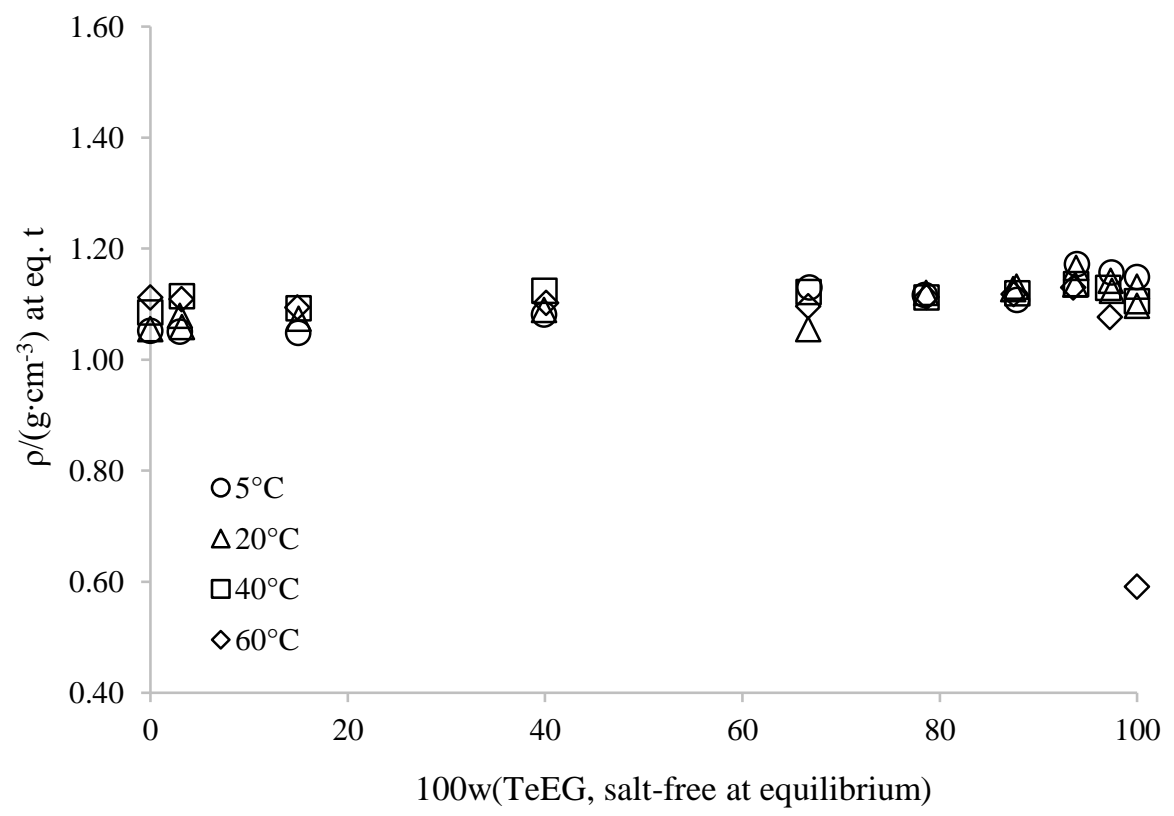

Figure 14: Density of saturated $\mathrm{NaHCO}_{3}-\mathrm{TeEG}-\mathrm{H}_{2} \mathrm{O}$ solutions measured at selected isotherms at atmospheric pressure. 


\section{Conclusion}

The solubility of $\mathrm{NaHCO}_{3}$ and the density of saturated mixtures of $\mathrm{MEG}-\mathrm{H}_{2} \mathrm{O}, \mathrm{DEG}-\mathrm{H}_{2} \mathrm{O}$, TEG$\mathrm{H}_{2} \mathrm{O}$, and TeEG- $\mathrm{H}_{2} \mathrm{O}$ were measured in this work. Potentiometric titration and the reverse Schreinemakers method were used to analyse and calculate the solubility.

For all systems, minima were found at $x$ (glycol, salt-free $)=0.5$. The minima remained constant at all temperatures. Two possible types of $\mathrm{NaHCO}_{3}$ solvation exist in the systems: 1) between water and $\mathrm{NaHCO}_{3}$ and 2) between glycol and $\mathrm{NaHCO}_{3}$. The affinity for $\mathrm{NaHCO}_{3}$ was apparently equally shared by water and glycol. The bonds between the solvent molecules is apparently stronger than to the ions of $\mathrm{NaHCO}_{3}$.

The density was measured by knowing the volume and weight of a sample. The accuracy of this method is very low, giving standard deviations up to $2 \%$. The trend of the density values was similar to the trend of the solubility values. Minima were located in the range $w$ (glycol, salt-free) 80 to $90 \mathrm{wt} \%$ glycol, salt-free.

The experimental data produced in this work can be used to enhance the quality of modelling of corrosion and gas drying. Furthermore the data can be used as a general test-bed for groupcontribution thermodynamic modelling of electrolyte system.

\section{AUTHOR INFORMATION}

\section{Corresponding Author}

*Tel.: + 454525 2868. E-mail address: plf@kt.dtu.dk 


\section{Author Contributions}

The manuscript was written through contributions of all authors. All authors have given approval to the final version of the manuscript.

\section{Funding Sources}

The Financial support from the Center for Energy Resources Engineering (CERE).

\section{Acknowledgement}

This work was performed in collaboration with lab technician Sandy Clayton.

\section{ABBREVIATIONS}

DEG, diethylene glycol; IR, infrared; MEG, monoethylene glycol; PE, polyethylene; RS, reverse Schreinemakers; TeEG, tetraethylene glycol; TEG, triethylene glycol; XRPD, x-ray powder diffraction.

\section{REFERENCES}

(1) Xie, Y.; Xu, L.; Gao, C.; Chang, W.; Lu, M. Corrosion Behavior of novel 3\% Cr pipeline steel in CO2 Top-of-Line Corrosion environment. Mater Des. 2011, 36, 54-57.

(2) Kermani, M. B.; Morshed, A. Carbon dioxide Corrosion in Oil and Gas Production - A Compendium. Corrosion. 2020, 59, 659-683.

(3) Farelas, F.; Galicia, M.; Brown, B.; Nesic, S.; Castaneda, H. Evolution of Dissolution processes at the interface of carbon steel corroding in a $\mathrm{CO} 2$ environment studied by EIS. Corros Sci. 2009, 52, 509-517. 
(4) Fosbøl, P. L. Carbon Dioxide Corrosion: Modelling and Experimental Work Applied to Natural Gas Pipelines. Ph.D thesis, Technical University of Denmark, 2007.

(5) Dugstad, A.; Lunde, L.; Nesic, S. Control of internal corrosion in multi-phase oil and gas pipelines. Prevention of Pipeline Corrosion Conference. 1994.

(6) Hammerschmidt, E. G. Formation of Gas Hydrates in Natural Gas Transmission Lines. Ind Eng Chem. 1934, 26, 851-855.

(7) Masoudi, R.; Tohidi, B.; Danesh, A.; Todd, A. C.; Anderson, R.; Burgass, R. W.; Yanga, J. Measurement and prediction of gas hydrate and hydrated salt equilibria in aqueous ethylene glycol and electrolyte solutions. Chem Eng Sci. 2005, 60, 4213-4224.

(8) Wu, M.; Wang, S.; Liu, H. A Study on Inhibitors for the Prevention of Hydrate Formation in Gas Transmission Pipeline. J Nat Gas Chem. 2007, 16, 81-85.

(9) Dugstad, A.; Seiersten, M. pH-stabilisation, a reliable method for corrosion control of wet gas pipelines. Proc - First Int Oilf Corros Symp Manag Corros Oil Gas Prod Fluids, Chem Mater More. 2004, 83-90.

(10) Kohl A. L.; Nielsen, R. B. Gas Purification. Fifth edit. Houston, Texas: Gulf Publishing Company, 1997.

(11) Fosbøl, P. L.; Thomsen, K.; Stenby, E. H. Solubility measurements in the mixed solvent electrolyte system Na2CO3-NaHCO3-monoethylene glycol-water. Ind Eng Chem Res. 2009, 48, 2218-2228. 
(12) Gärtner, R. S.; Seckler, M. M.; Witkamp, G-J. Solid Phases and Their Solubilities in the System Na2CO3 + NaHCO3 + Ethylene Glycol + Water from (50 to 90) ${ }^{\circ} \mathrm{C}$. Engineering. 2004, $3,116-125$.

(13) Sandengen, K. Prediction of Mineral Scale Formation in Wet Gas Condensate Pipelines and in MEG (Mono Ethylene Glycol) Regeneration Plants. Ph.D. thesis, NTNU, 2006.

(14) Fosbøl, P. L; Thomsen, K.; Stenby, E. H. Reverse Schreinemakers method for experimental analysis of mixed-solvent electrolyte systems. J Solution Chem. 2009, 38, 1-14.

(15) Kaasa, B.; Sandengen, K.; Østvold, T. Thermodynamic Predictions of Scale Potential, pH, and Gas Solubility in Glycol-Containing Systems. SPE Symposium, Aberdeen, UK. , 2005, 133145.

(16) Toporescu, E. Sur la preparation du bicarbonate du sodium. Compt Rend. 1922, 175, 268270.

(17) Freeth, F. A. The system: $\mathrm{Na} 2 \mathrm{O}-\mathrm{CO} 2-\mathrm{NaCl}-\mathrm{H} 2 \mathrm{O}$, considered as two four- component systems. Phil Trans Roy Soc(London), ser A. 1922, 223, 35-87.

(18) Fedotieff, P. P. Der Ammoniaksodaprozes vom standpunkte der phasenlehre. Z Phys Chemie. 1904, 49, 162-188.

(19) Trypuc, M. Kielkowska, U. Solubility in the NH4HCO3 $+\mathrm{NaHCO} 3+\mathrm{H} 2 \mathrm{O}$ System. $J$ Chem Eng Data. 1998, 43, 201-204.

(20) Ellingboe, J. L.; Runnels, J. H. Solubilities of Sodium Carbonate and Sodium Bicarbonate in Acetone-Water and Methanol-Water Mixtures. J Chem Eng Data. 1966, 11, 323-324. 
(21) Bogoyavlensky, P. S.; Manannikova, A. S. The NaCl-NaHCO3-H2O system (Carlsbad salt) at 25 and $38^{\circ} \mathrm{C} . J \mathrm{Appl}$ Chem USSR. 1955, 28, 225-228.

(22) Bekturov, A. B.; Naimushina, R. F.; Konobritskii, E. G.; Litvinenko V. I. Physicochemical studies of the processing of natural borates. Tr Instituta Khimicheskikh Nauk Akad Nauk Kazakhskoi SSR. 1967, 16, 137-165.

(23) Toporescu, E. Sur la preparation du bicarbonate du sodium. Compt Rend. 1922, 174, 870873.

(24) Neumann, B.; Domke, R. Die Gleichgewichtsverhältnisse beim ammoniaksodaprozesse unter druck. Z Elektrochem. 1928, 34, 136-153.

(25) Ponizovskii, A. M.; Vladimirova N. M. Solubility of the system Na, Mg, Cl, HCO3 - H2O. Proc Acad Sci USSR, Sect Chem Dokl Akad Nauk SSSR. 1959, 126, 345-346.

(26) Luzhnaya, N. P.; Kosyachkova, S. N. The solubility isotherm of the aqueous system $2 \mathrm{NaHCO} 3+\mathrm{K} 2 \mathrm{SO} 4<->2 \mathrm{KHCO} 3+\mathrm{Na} 2 \mathrm{SO} 4$ at 50 ${ }^{\circ} \mathrm{C}$. Izv Sektora Fiz-Khim Anal, Inst Obshch Neorgan Khim Akad Nauk. 1956, 27, 358-366.

(27) Makarov, S. Z.; Vaksberg, N. M. A study of equilibrium in soda-lake brines. Zh Russ FizKhim Obs. 1930, 62, 1870.

(28) Hill, A. E.; Bacon, L. R. Ternary Systems VI. Sodium Carbonate, Sodium Bicarbonate and Water. J Am Chem Soc. 1927, 49, 2487-2495.

(29) Wegscheider, R.; Mehl J. Über Systeme Na2CO3-NaHCO3-H2O und das existenzbebiet der Trona. Monatshefte Chem /Österreichische Akad der Wissenschaften. 1928, 49, 283-315. 
(30) Sedel'nikov, G.S.; Lazareva, A. I. Analysis of solid, liquid, and gaseous phases in carbonate/bicarbonate system. Inst Obs Neorgan Khim Akad Nauk SSSR. 1948, 14, 1176-1178.

(31) Bogoyavlenskii, P. S.; Shih-Ching, N. Solubility relation in the system NaHCO3-KHCO3$\mathrm{H} 2 \mathrm{O}$ and its dependence on some peculiarities of the structure of the salts. Sci Sin (English Ed. $1959,8,580-590$.

(32) Gärtner, R. S.; Seckler, M. M. Witkamp GJ. Reactive recrystallization of sodium bicarbonate. Ind Eng Chem Res. 2005, 44, 4272-4283.

(33) Corradini, F.; Marcheselli, L.; Tassi, L.; Tosi, G. Ethane-1,2-diol-water solvent system: Relative permittivity as a function of temperature and binary composition. J Chem Soc Faraday Trans. 1993, 89, 123-127.

(34) Masoudi, R.; Tohidi, B.; Anderson, R.; Burgass, R. W.; Yang, J. Experimental measurement and thermodynamic modelling of clathrate hydrate equilibria and salt solubility in aqueous ethylene glycol and electrolyte solutions. Fluid Phase Equilib. 2004, 219, 157-163.

(35) Vener, R. E.; Thompson, A. R. Solubility and Density Isotherms for Sodium SulfateEthylene Glycol-Water. Ind Eng Chem. 1949, 41, 2242-2247. 
For Table of Contents Only

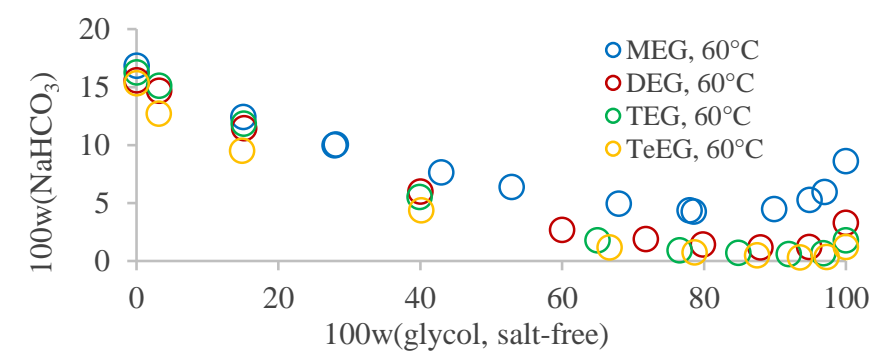

